\title{
Establishing the link between habitat selection and animal population dynamics
}

\author{
Jason Matthiopoulos, ${ }^{1,7}$ John Fieberg, ${ }^{2}$ Geert Aarts, ${ }^{3,4}$ Hawthorne L. Beyer, ${ }^{5}$ Juan M. Morales, ${ }^{6}$ \\ AND DANIEL T. HAYDON ${ }^{1}$ \\ ${ }^{1}$ Institute of Biodiversity, Animal Health and Comparative Medicine, College of Medical, Veterinary, and Life Sciences, \\ Graham Kerr Building, University of Glasgow, Glasgow G128QQ United Kingdom \\ ${ }^{2}$ Department of Fisheries, Wildlife and Conservation Biology, University of Minnesota, St. Paul, Minnesota 55108 USA \\ ${ }^{3}$ IMARES Wageningen UR, Institute for Marine Resources and Ecosystem Studies, \\ P.O. Box 167, 1790 AD Den Burg, The Netherlands \\ ${ }^{4}$ Department of Aquatic Ecology and Water Quality Management, Wageningen UR, P.O. Box 47, \\ 6700AA Wageningen, The Netherlands \\ ${ }^{5}$ ARC Centre of Excellence for Environmental Decisions, Centre for Biodiversity \& Conservation Science, \\ University of Queensland, Brisbane, Queensland 4072 Australia \\ ${ }^{6}$ Laboratorio ECOTONO, INIBIOMA-CONICET, Universidad Nacional del Comahue, Quintral 1250, (8400) Bariloche, Argentina
}

\begin{abstract}
Although classical ecological theory (e.g., on ideal free consumers) recognizes the potential effect of population density on the spatial distribution of animals, empirical species distribution models assume that species-habitat relationships remain unchanged across a range of population sizes. Conversely, even though ecological models and experiments have demonstrated the importance of spatial heterogeneity for the rate of population change, we still have no practical method for making the connection between the makeup of real environments, the expected distribution and fitness of their occupants, and the long-term implications of fitness for population growth. Here, we synthesize several conceptual advances into a mathematical framework using a measure of fitness to link habitat availability/selection to (density-dependent) population growth in mobile animal species. A key feature of this approach is that it distinguishes between apparent habitat suitability and the true, underlying contribution of a habitat to fitness, allowing the statistical coefficients of both to be estimated from multiple observation instances of the species in different environments and stages of numerical growth. Hence, it leverages data from both historical population time series and snapshots of species distribution to predict population performance under environmental change. We propose this framework as a foundation for building more realistic connections between a population's use of space and its subsequent dynamics (and hence a contribution to the ongoing efforts to estimate a species' critical habitat and fundamental niche). We therefore detail its associated definitions and simplifying assumptions, because they point to the framework's future extensions. We show how the model can be fit to data on species distributions and population dynamics, using standard statistical methods, and we illustrate its application with an individual-based simulation. When contrasted with nonspatial population models, our approach is better at fitting and predicting population growth rates and carrying capacities. Our approach can be generalized to include a diverse range of biological considerations. We discuss these possible extensions and applications to real data.
\end{abstract}

Key words: accessibility; climate change; conservation; density dependence; generalized functional response; generalized linear model; habitat suitability; ideal free distribution; mathematical model; resource selection function; simulation; species distribution models.

\section{INTRODUCTION}

Accelerating environmental change requires us to understand not just how species distributions will adjust, but also whether their population sizes will go up or down. The mechanisms linking the environment of a population to its spatial distribution and growth are considered textbook material (e.g., Chapman and Reiss 1999, Begon et al. 2006, Levin 2009). Environmental

Manuscript received 24 November 2014; accepted 18 December 2014; final version received 12 January 2015. Corresponding Editor: B. E. Kendall.

${ }^{7}$ E-mail: Jason.Matthiopoulos@glasgow.ac.uk variables are distributed across space, their combinations forming habitats that are differentially used by different species. Populations track the heterogeneity in their environment either actively (when individuals navigate the landscape in search of suitable habitats) or passively (when dispersers settle at habitats that differentially affect their survival and reproduction). These processes are tightly linked: increases in population density will tend to lower local fitness and, additionally, cause some individuals to move to suboptimal habitats, directly impacting the overall ability of a population to grow (Fig. 1). 


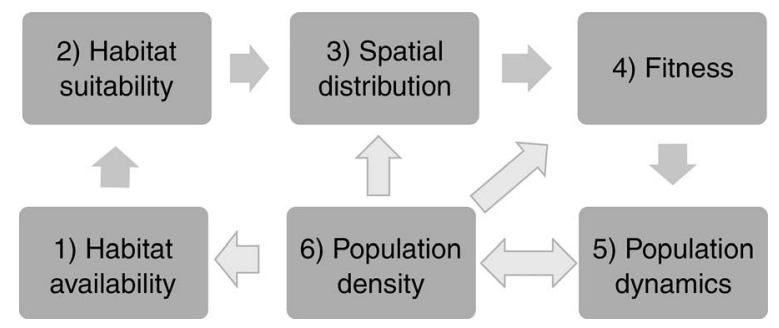

FIG. 1. Conceptual links between the makeup of the environment and the dynamics of a population that lives in it. Habitat availability (1) describes the amounts of all habitats that are accessible to an organism. The suitability of different habitats (2) gives rise to an organism's spatial distribution (3). Across different locations, organisms may experience different conditions, access different amounts of resources, and be subjected to different degrees of risk. These experiences of individuals determine their fitness (4). The collective measure of fitness for the entire population determines annual rates of population growth and subsequent population dynamics (5). Population density (6) then feeds back into population dynamics, but it may also affect habitat availability (through resource depletion or niche engineering), spatial distribution (through behavioral responses to crowding), and fitness (through demographic responses to crowding). Note that the suitability of different habitats is a characteristic of a species and therefore cannot be changed by population density.

Much of the ecological theory developed over past decades has focused on different stages of this causal chain: reaction-diffusion models (Solé and Bascompte 2006, Okubo and Levin 2010) have explored the emergence of complex spatial patterns, optimal foraging (MacArthur and Pianka 1966, Emlen 1968) has determined the behavioral strategies that maximize energy intake (indirectly highlighting the limits of environmental profitability for the individual), ideal free distribution theory (Fretwell and Lucas 1970) has opened the debate about the implications of competition for the distribution of conspecifics, movement ecology (Turchin 1998, Codling et al. 2008, Schick et al. 2008) has created useful abstractions to describe how organisms navigate their environment, behavioral ecology (Krebs and Davies 1997) has increasingly considered energetic and riskrelated trade-offs in trying to explain the decisions of individuals from a fitness perspective, and population ecology persistently aims to incorporate the implications of individual fitness for demographic rates and net population growth (Clutton-Brock et al. 1991, Silvertown et al. 1993, Gaillard et al. 2000, Clutton-Brock and Coulson 2002, Coulson et al. 2005, Matthiopoulos et al. 2014).

Yet, despite our ability to enumerate the links in the chain that ties a population to its environment, and despite the multitude of theoretical insights obtained since the 1960s, we still lack practicable models with estimable parameters that can capture species-environment relationships in an integrated spatial and temporal fashion (Keith et al. 2008).

Statistical species distribution models (SDMs) have thrived in the last 25 years because of the emergence of new data-collection technologies (Millspaugh and Marzluff 2001, Cagnacci et al. 2010) and data analysis techniques (Buckland and Elston 1993, Boyce and McDonald 1999, Guisan and Zimmermann 2000, Guisan et al. 2002, Manly et al. 2002, Scott et al. 2002, Aarts et al. 2008). Despite their bewildering variety (Guisan and Zimmermann 2000, Elith and Leathwick 2009), fundamentally different assumptions, and regularly conflicting outputs (Elith and Graham 2009), the basic thrust of these models is the same: organisms have a reason for being where we find them. They are observed at (or near) places that, in some way, help them survive and reproduce. By using one of several possible quantitative methods that can correlate the distribution of species observations (i.e., counts or occurrences) to different environmental gradients, SDMs hope to empirically capture enough of the spatial signal to further our ecological understanding of the species. The predictions from these models are used for interpolating patchy spatial data, expanding the spatial range of available species maps, or anticipating redistribution under environmental change.

Habitat selection functions (HSFs, more widely referred to in the literature as resource selection functions; Boyce and McDonald 1999, Manly et al. 2002) are arguably the best-established and bestunderstood type of SDM. A HSF is often defined as any model that yields values proportional to usage. More precisely, a HSF models the expected density (i.e., the intensity) of observations as a function of covariates (Aarts et al. 2012). At their simplest, they are implemented as generalized linear models (GLMs; McCullagh and Nelder 1989), with more recent extensions such as generalized additive (GAMs; Wood 2006), or mixed-effects models (GLMMs or GAMMs; Pinheiro and Bates 2000, Wood 2006) aimed at capturing nonlinear responses to the environment that are affected by multiple sources of variability. HSFs are supported by extensive statistical theory, widely available software, and graphical diagnostics, and they frequently outperform more opaque machine-learning methods such as neural nets (Wenger and Olden 2012). Furthermore, they are more general than (although conceptually related to) more recent, popular methods such as maximum entropy (Phillips et al. 2006, Elith et al. 2011, Aarts et al. 2012, Renner and Warton 2013). Therefore, HSFs are a solid foundation upon which to start developing the empirical link between habitat use and population dynamics (McLoughlin et al. 2010).

However, both the deductive and predictive abilities of SDMs have come under severe criticism. The phenomenological origin of their mathematical structure has encouraged the proliferation of ad hoc methodological variants, impeding the biological interpretation of model structure and results (Elith and Leathwick 2009). Their misleading use as descriptors of a species' realized/fundamental niche (Stockwell 2007, Hirzel and Le Lay 2008) has received negative and recurrent 
attention (Elith and Leathwick 2009, Soberón and Nakamura 2009, Peterson et al. 2011, Warren 2012, 2013, McDonald et al. 2013, McInerny and Etienne 2013). Their sensitivity on arbitrary scale decisions made by the analyst (Austin 1999, Beyer et al. 2010) and instability in changing environments (Randin et al. 2006, Zurell et al. 2009, McLoughlin et al. 2010, Sinclair et al. 2010, Matthiopoulos et al. 2011, Wenger and Olden 2012) has alerted practitioners to the dangers of their widespread and unvalidated application. Additionally, a crucial fact that seems to have escaped the attention of the SDM literature is that estimates of habitat suitability are not likely to be invariant to population density. Given that these models evaluate habitat suitability on the basis of relative usage, which is certain to be affected by density-dependent processes, SDMs run the risk of returning different parameter estimates depending on how close an observed population is to its carrying capacity.

Despite the development of more mechanistic spatial approaches (Chase and Leibold 2003, Kearney and Porter 2004, Moorcroft and Lewis 2006, Patterson et al. 2008, Schick et al. 2008, Higgins et al. 2012), empirical SDMs are unparalleled in their taxonomic generality, ease of use, and computational expediency for population-level inferences. For this reason, there is a concerted remedial effort to try and improve their shortcomings (Arthur et al. 1996, Mauritzen et al. 2003, Gillies et al. 2006, Hebblewhite and Merrill 2008, Godvik et al. 2009, McLoughlin et al. 2010, Matthiopoulos et al. 2011) and help them fulfill their original deductive and predictive promise (Warren 2013). In this study, we propose a pragmatic synthesis between models of habitat selection and models of population change. Our overarching objective is to mathematically link empirical estimates of habitat availability and apparent habitat preference with the observed rates of growth of populations living in these environments. We believe that such a synthesis can ultimately lead to both a deeper mechanistic understanding and stronger statistical inference. Therefore, to illustrate the utility of our paradigm, we take the first steps along both of these routes. On the mechanistic side, we explore how apparent habitat suitability, gleaned through observations of space use, may be connected to the unobserved fitness that animals gain from each habitat. On the statistical side, using simulated data, we show how inference on spatial usage and population time series can improve our predictions of population change.

Since we aim for a convergence between the relatively independent areas of species distribution modeling and population dynamics, we begin by explaining our terminology, which borrows vocabulary from both areas. We limit our attention to a specific, but still quite broad set of circumstances that we believe can serve both as proof of concept and as a suitable basis for expansion. Hence, in Ecological scope and simplifying assumptions, we set out the basic premises of our study.
In Environmental determinants of fitness, we outline a general link between fitness and the environment of an organism and in Linking fitness, habitat suitability, and habitat use, we introduce the connection between fitness, habitat suitability, and space use. In Parametric formulations of habitat availability, we import, from the statistical literature, methodology that can abstract the composition of environmental space, reducing the detailed availability profile for all habitats to a simple parametric approximation. This approximation allows us to obtain computationally efficient expressions for the fitness of organisms living in different environments under exponential (Connecting habitat use to exponential population growth) and density-dependent (Connecting habitat use to density-dependent population growth) population growth. In A note on the relationship between partial fitness and habitat suitability, we discuss the link between measures of habitat suitability (derived from observations of habitat usage) and the underlying fitness offered by different habitats. In Parameter estimation from space-use and population time-series data, we describe how the analytical expressions from Connecting habitat use to exponential population growth and Connecting habitat use to density-dependent population growth can be used with real data on population distribution and growth to estimate the parameters linking the environment to the average fitness of a population. We apply these methods to data from a simulation of animal redistribution and demography (described in Simulation) in three simulation experiments (outlined in Simulation experiments) that examine the goodness of fit of the method, its predictive ability, and its sensitivity to the amount and type of available data. Finally, we discuss how the work presented here can be extended, hence outlining a research program that aspires to the development of practitioner-friendly joint inference from spatial and temporal data.

\section{TERMinOLOGY}

We retain the basic distinction between geographical space ( $G$-space) and environmental space ( $E$-space), historically known as Hutchinson's duality (Hirzel and LeLay 2008, Colwell and Rangel 2009, Elith and Leathwick 2009). $G$-space comprises the three dimensions of latitude, longitude, and altitude/depth, often projected onto a Cartesian system of coordinates. In contrast, each dimension in $E$-space represents a biotic or abiotic environmental variable, i.e., a continuous, discrete, or qualitative random variable representing a condition (e.g., pH, temperature, sea depth), resource (e.g., soil nutrients, prey, breeding sites), or perceptible threat (e.g., predators, pollution). Environmental variables may or may not correlate with the geographic distribution of the study species. Those that do are called its covariates.

Here and elsewhere (Aarts et al. 2008, Matthiopoulos et al. 2011), we define a habitat $\mathbf{x}$ as a point in $E$-space, the combination of specific values for $K$ environmental 
variables (e.g., geomorphology and climate variables combining into the characteristic makeup of polar habitat). Alternatively, habitat has been defined in a species-dependent way as the region in geographical space in which an organism lives (e.g., polar bear habitat). The two definitions are not interchangeable (see Hall et al. 1997). We opt for the first definition because it allows objective comparisons between species and quantitative gradations of suitability.

Space use $\left(u_{\mathrm{s}}\right)$ is the expected usage of the neighborhood (e.g., a grid cell) centered at a point $\mathbf{s}$ in $G$-space, i.e., the proportion of an individual's or a population's time that is likely to be spent there on average. Use can be equally well defined infinitesimally on single points in space as the spatially varying intensity function of an inhomogeneous Poisson process (Warton and Shepherd 2010, Aarts et al. 2012). Habitat use $\left(u_{\mathbf{x}}\right)$, the proportion of time spent in regions of $E$-space, or equivalently, the intensity of use of points in $E$-space, is not only influenced by the suitability of these habitats to an organism, but also by the abundance and accessibility (Matthiopoulos 2003) of these habitats (collectively, their availability). Assuming purely continuous environmental variables (with no loss of generality), we introduce the function $f_{\mathbf{x}}$ representing habitat availability as the probability density of habitat $\mathbf{x}$ in $E$-space (i.e., the unconditional likelihood with which this habitat occurs at any given point of $G$-space).

If the behavior and demography of organisms were unaffected by their environment and they were allowed to move/disperse randomly for a long time within the study area (resulting in an asymptotically homogeneous distribution in $G$-space), habitats would be used in proportion to their availability. Therefore, deviations from proportional usage indicate the existence of a response (apparent preference or avoidance). Consequently, many analyses define preference $w_{\mathbf{x}}$ as proportional to the ratio of habitat use over availability (Johnson 1980, Manly et al. 2002, Aarts et al. 2008, 2013). Different animals will vary in the behavioral perceptiveness, speed, and precision with which they can track good habitats in the environment. The presence of an organism in a particular habitat may be as much the result of active selection as of passive happenstance (an individual may be encountered there because of differential survival rather than choice). Here, we will replace the active term "habitat preference" by the more passive "apparent habitat suitability".

The average fitness $F(\mathbf{f})$ that a population can acquire from its environment (denoted $\mathbf{f}$, the vector of individual availabilities $f_{\mathbf{x}}$ for all habitats $\mathbf{x}$ in $E$-space ) is defined as the population's log-rate of change (we will expand on this definition of fitness in Environmental determinants of fitness). A habitat that can satisfy all life-history priorities of a species (e.g., nutrition, rest, mating, birth) may be called sufficient. A habitat that can satisfy only part (or none) of the life-history priorities is called insufficient. We will call multifunctional those habitats that can satisfy more than one life-history priority. Sufficient habitats are multifunctional but the reverse may not be true. We define partial fitness $F_{\mathbf{x}} \in(-\infty, \infty)$ as the contribution of each unit of habitat $\mathbf{x}$ to the average fitness of a population. Partial fitness can be interpreted as the fitness of a population living in an environment made up entirely of habitat type $\mathbf{x}$.

\section{Ecological Scope and Simplifying Assumptions}

We collect here 10 important assumptions that set out the scope of this study. Relaxing these assumptions will form the basis for future extensions of our work, so we return to them in Discussion.

1. Accessibility of environment to the population.-An assumption implicitly made by most analyses of species distribution is that the populations are not systematically (or due to historical effects) prevented from accessing good-quality habitats (Manly et al. 2002, Matthiopoulos 2003). This assumption could, for example, be violated by natural or manmade boundaries (Beyer et al. 2014), or by the existence of transient processes such as invasion fronts. In practice, this requires the user to define an appropriate $G$-space in which all areas are accessible by the population (Northrup et al. 2013). For our simulations (Simulation), we reduce the effects of accessibility by implementing toroidal spatial boundaries and employing a settlement phase at the start of each simulation.

2. Spatial pseudo-equilibrium.-Our assumption here (as in most SDM approaches; Guisan and Thuiller 2005) is that the spatial usage data do not capture a population whose distribution is still undergoing changes due to a delayed response to the environment. In the context of a continually changing environment, we assume that the spatial distribution of a population adjusts readily, and thus, SDMs fitted to annual data are not likely to be misguided by transient patterns. This assumption builds on assumption 1 (on accessibility) by requiring that all of space is accessible by the population rapidly enough to allow the pseudo-equilibrium distribution to be approximately achieved between sampled snapshots of spatial distribution. Note that this does not require the population size to have reached equilibrium.

3. Habitat use by individuals is representative of the population's habitat use.-Our methods, as they currently stand, apply to freely moving animals whose survival and reproductive success depend on all of the habitats they have experienced during a year. We assume that any population member experiences and uses approximately the same mix of available habitats as all the others. This assumption need not require accessibility of all the landscape by every single individual, if the environmental composition at the scale of movement of individuals is sufficiently representative of the composition of the entire landscape.

4. Treatment of resource depletion.-Although our model examines the effect of limited resources on generating density dependence, for this study, we have 
not examined the impact of the focal species on the resources it relies on. Our framework can, in principle, be extended to model resource-consumer dynamics (by running coupled dynamical models for more than one species), but we have here focused on the single-species case. We justify this assumption by distinguishing between three possibilities. For some species (e.g., generalist consumers), resources may regenerate quickly, or their abundance may be driven by processes other than the focal species' density. In such a scenario, any process of depletion will not be strongly coupled with the dynamics of the study species. If the species of interest causes slow depletion (e.g., over multiple years), then this effect can be represented by snapshot data on the annual distribution of the resources that enter the model as a covariate. If depletion exists and is fast, a resource layer can be depleted within the interval of a year, potentially removing any initial spatial heterogeneity in its distribution. An SDM trying to correlate species distribution with such a depleted resource would usually fail to find a signal. This is a recognized issue in the SDM literature and one advantage of correlating usage with non-depletable environmental proxies of resource productivity, instead of data on resource abundance (Torres et al. 2008, Aarts et al. 2014).

5. Linearity in density dependence.-We have assumed that use of a particular spatial unit by each additional member of the population lowers that unit's suitability for the other individuals in it. This excludes processes such as the Allee effect, which would signify that, at low overall values of density, increases in density can have a positive effect on fitness (e.g., by alleviating the per capita effect of predation risk at larger populations). Although such a feature is not included here, it could be captured by higher-order (e.g., quadratic) terms in our model of density dependence.

6. Linearity in the response of fitness to increasing usage of habitats or to increases in individual resources.For mathematical simplicity, we here exclude the possibility of diminishing fitness benefits from the superabundance of any given habitat, or specific resource. This means that every additional unit of good habitat or resource has an unsaturating contribution to population growth. From an individual's point of view, the benefit obtained from increasing amounts of a resource or increasing usage of a particular habitat should plateau to an asymptotic maximum (Austin 2002), for example, due to satiation. However, from a population perspective, the number of individuals that can be sustained by an ever-increasing resource is unlimited. Hence, any short-term effects (such as the daily satiation of an individual) should be counterbalanced by high survival and increases in the production of new individuals.

7. Additivity of covariates in determining partial fitness (within a habitat).-Although it is certainly true that different resources, conditions, or sources of risk may interact nonlinearly (in a complementary or antagonistic way) in determining organism fitness (Tilman 1982), this important extension is beyond the scope of the present study. We will instead model these effects as purely additive influences within the linear predictor of our statistical model of fitness (see Eq. 4).

8. Additivity of partial fitness in determining average fitness (across different habitats).-We will assume that the use of different habitats has an additive effect on fitness. For example, this implies that organisms cannot construct sufficient habitats by complementary use of insufficient ones.

9. No population structure.-The population models examined in this study are simple. Beyond the focal features of density dependence and spatial/habitat effects, we have eschewed the possibility of reproductive time delays, age structure, or any other form of individual variation that is not driven by habitat.

10. No genetic change.-Assumption 9 also implies that no evolutionary processes take place. Hence, we require that the study questions are posed over short time-horizons, population members are genetically similar, and any environmental change is sufficiently non-directional that the only adaptation takes the form of spatial redistribution.

\section{Environmental Determinants of Fitness}

In our approach, the fundamental link between spatial distribution and population dynamics is the average fitness of a population. Fitness formalizes the intuitive notion that different environments (i.e., collections of habitats) should differentially affect a population's shortterm rate of growth and long-term size (its carrying capacity). In general, the fitness $F\left(\mathbf{f}, N_{t}\right)$ that a population can acquire from a given environment $\mathbf{f}=\left\{f_{\mathbf{x}}\right.$, for every habitat $\mathbf{x}$ in $E\}$ is defined as the population's log-rate of change, given its current size $\left(N_{t}\right)$

$$
\frac{N_{t+1}}{N_{t}}=\exp \left(F\left(\mathbf{f}, N_{t}\right)\right)
$$

We will further expand on issues of density dependence in Connecting habitat use to density-dependent population growth, and focus here on the dependence of fitness on environmental makeup. Discrete-time models are used because they offer an easier entry point for empirical investigations (population data are likely to be discrete in time), but the same ideas could be couched in continuous time. This ecological interpretation of Eq. 1 has been extensively discussed in the literature (Stenseth 1983, 1984, Nur 1984, 1987, Murray 1985, Ollason 1991, Mills 2012) and the equation has a long history of use in evolutionary models (Fisher 1930, Lande 1982, Roff 2008). Due to the exclusion of genetic adaptation (assumption 10), the interpretation of fitness in this study is purely ecological, not evolutionary. Hence, our framework currently builds no link between evolutionary fitness (the viability of a particular genotype living in a 
constant environment, a situation where genotypes are variable in time and between individuals) and population fitness (looking at collective viability in a particular environment, a situation where environments change either due to shifts in habitat availability or due to intra/interspecific processes).

Because partial fitness is here assumed additive (assumption 8) and each additional unit of usage received by habitat $\mathbf{x}$ is postulated to have an undiminishing contribution to average fitness (assumption 6), partial fitness $\left(F_{\mathbf{x}}\right)$ scales with the usage $u_{\mathbf{x}}$ of each habitat to give the relationship between average and partial fitness

$$
F=\int_{E} F_{\mathbf{x}} u_{\mathbf{x}} d \mathbf{x}
$$

This last expression derives expected population fitness as the average (over $E$-space) of habitat-specific fitness, weighted by habitat-specific usage (an illustrated example of this concept is presented in Fig. 2).

Assumption 3 ensures that placing this in Eq. 1 yields a per capita growth rate that is representative of the whole population. The two components of Eq. $2\left(F_{\mathbf{x}}\right.$ and $\left.u_{\mathbf{x}}\right)$ are now expanded individually here and in Linking fitness, habitat use, and habitat suitability.

Partial fitness can be specified further by considering its sign in relation to the type of habitat that it represents

$$
F_{\mathbf{x}}\left\{\begin{array}{l}
<0 \text { if } \mathbf{x} \text { is insufficient } \\
=0 \text { if } \mathbf{x} \text { is fitness-neutral } \\
>0 \text { if } \mathbf{x} \text { is sufficient }
\end{array}\right.
$$

Further progress can be made by considering a simple classification of environmental variables into (1) resources $(R),(2)$ risks $(P)$, and (3) conditions $(C)$. Resources are environmental variables whose density has a positive and monotonic relationship with fitness (i.e., the more, the better). Resources can potentially be depleted (locally or globally) by an organism, although we will not explicitly consider such processes here (assumption 4). In contrast, risks have a negative (but still monotonic) relationship with fitness. Conditions are environmental variables that characterize suitable habitat according to a bounded range of values. Like resources, they can potentially be altered by organisms (see literature on ecosystem engineers, e.g., OdlingSmee et al. [2003]). Hence, resources should push $F_{\mathbf{x}}$ above zero, sources of risk should push it below zero, and conditions could act in both ways (depending on whether their particular values are favorable or not). This classification is not unlike the one recommended by Guisan and Zimmermann (2000), Huston (2002), and Guisan and Thuiller (2005), however, here, we have justified the subdivision on the basis of the response of fitness to environmental gradients (increasing, decreasing, non-monotonic). In general, we can therefore write

$$
F_{\mathbf{x}}=\underbrace{\sum_{k=1}^{n_{1}} R\left(x_{k}\right)}_{\text {Resources }}+\underbrace{\sum_{k=n_{1}+1}^{n_{1}+n_{2}} P\left(x_{k}\right)}_{\text {Risks }}+\underbrace{\sum_{k=n_{1}+n_{2}+1}^{n_{1}+n_{2}+n_{3}} C\left(x_{k}\right)}_{\text {Conditions }}
$$

where the contribution of each of the three categories of fitness covariates may be determined according to a different functional form. Since here we ignore the possibility of interaction terms (assumption 7), for the rest of this study, we focus on a simplification of Eq. 4 that models the contributions to fitness by first- or second-order polynomials, so that

$$
\begin{aligned}
& R\left(x_{k}\right)=-\alpha_{0, k}+\alpha_{1, k} x_{k} \\
& P\left(x_{k}\right)=-\alpha_{1, k} x_{k} \\
& C\left(x_{k}\right)= \pm \alpha_{0, k} \pm \alpha_{1, k} x_{k}-\alpha_{2, k} x_{k}^{2}
\end{aligned}
$$

where the alphas are positive coefficient values forming the polynomials. These bear some biological interpretation. For example, if the $k$ th variable is a resource, then $\alpha_{0, k}$ is the rate at which fitness is lost when that resource is absent $\left(x_{k}=0\right)$. If the $k$ th variable is a risk, then $\alpha_{0, k}$ is set identically to zero, so that fitness is only lost under non-zero values of the variable $x_{k}$. $C\left(x_{k}\right)$ describes fitness arising from an environmental condition as a downward-pointing parabola with a maximum $\left( \pm \alpha_{0, k}+\alpha_{1, k}^{2} / 4 \alpha_{2, k}\right)$ at intermediate values and guaranteed negative fitness at sufficiently extreme (large and small) values of the environmental condition. Although it is possible that the response to conditions is asymmetric around the optimum (Austin 1999, 2002), a parabolic form is nevertheless a considerable improvement over simple linearity. Hence, this form seems a good compromise between biological realism and mathematical tractability. The reductions in fitness arising from sources of risk $P\left(x_{k}\right)$ take proportionately larger negative values for greater values of risk. This is an untested assumption, but we see no reason to doubt it from first principles. The linear response of fitness to resources $R\left(x_{k}\right)$ is anticipated by assumption 6 . Note that this discussion on the functional specification pertaining to conditions, resources, and risks merely aims to cast the simple empirical expression in Eq. 5 in a more biological light. For the implementation of the framework, it is not necessary for the user to classify different fitness covariates a priori (e.g., if unsure, all covariates can default to a second-order polynomial and the process of model estimation/selection can be relied upon to eliminate any unnecessary high-order terms). Hence, replacing the positive alphas (Eq. 5) by signed betas gives the more general form

$$
F_{\mathbf{x}}=\sum_{k=1}^{K} \sum_{r=0}^{2} \beta_{r, k} x_{k}^{r} \quad \beta_{2, k} \leq 0 .
$$




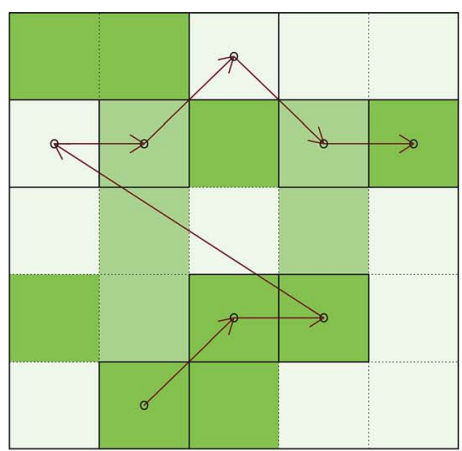

a) Habitat selection landscape

$$
\begin{aligned}
& f_{1}=\frac{11}{25} \\
& f_{2}=\frac{5}{25} \\
& f_{3}=\frac{9}{25}
\end{aligned}
$$

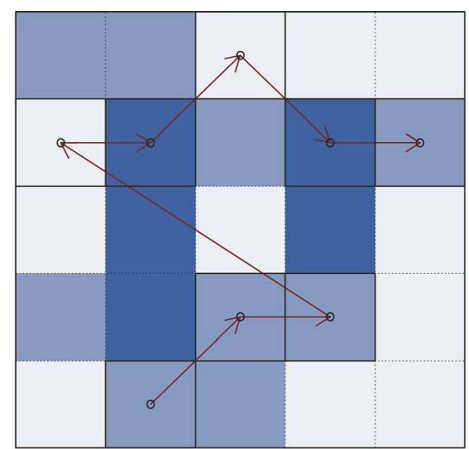

b) Partial fitness landscape

$$
\begin{aligned}
& F_{1}=-0.5 \\
& F_{2}=0.4 \\
& F_{3}=2.0
\end{aligned}
$$

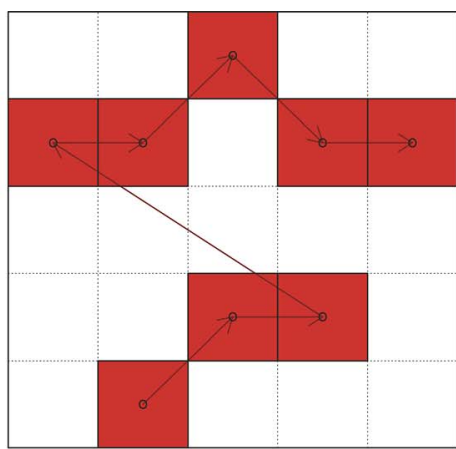

c) Usage landscape

$$
\begin{aligned}
& u_{1}=\frac{2}{8} \\
& u_{2}=\frac{2}{8} \\
& u_{3}=\frac{4}{8}
\end{aligned}
$$

$$
F=\sum_{i=1}^{3} F_{i} u_{i}=\left(-0.5 \frac{2}{8}+0.4 \frac{2}{8}+2 \frac{4}{8}\right)=0.975
$$

FIG. 2. A simplified illustration showing how population growth rate is assumed to arise from habitat usage. In (a), we envisage the movement (red arrows) of a single organism through a landscape of three habitat types (type 1 in white, type 2 in light green, and type 3 in dark green). The availability of each habitat type is denoted by $f_{i}$. Each habitat type has a different partial fitness contribution $F_{i}$, represented in (b) by white, light blue, and dark blue. As the organism moves through space, (c) its usage $u_{i}$ of different habitats is incremented each time a cell belonging to that habitat is visited. The aggregate fitness $F$ can then be calculated as an average of partial fitnesses, weighted by usage. If different individuals in the population are assumed to use a similar mix of the three habitats, then the individual's aggregate fitness can be applied to the population. In this example, the value is 0.975 , implying a per capita population growth of $\exp (F) \cong 2.65$.

\section{Linking Fitness, Habitat Suitability, and Habitat Use}

In this section, we derive a general expression (Eq. 10) linking average fitness to habitat suitability. This expression will then be used in Connecting habitat use to exponential population growth and Connecting habitat use to density-dependent population growth to derive exponential growth and density-dependent models, respectively. According to assumption 3, fitness is driven by an organism's usage of different habitats, in a way representative of the rest of the population. In general (Lele and Keim 2006, Aarts et al. 2012, Lele et al. 2013), habitat usage $\left(u_{\mathbf{x}}\right)$ is related to apparent habitat suitability $\left(w_{\mathbf{x}}\right)$ within the constraints of habitat availability $\left(f_{\mathbf{x}}\right)$

$$
u_{\mathbf{x}}=\frac{w_{\mathbf{x}} f_{\mathbf{x}}}{\int_{E} w_{\mathbf{y}} f_{\mathbf{y}} d \mathbf{y}} .
$$

Here, $\mathbf{y}$ is a dummy variable. This expression has been independently derived from several perspectives, most notably from weighted distribution arguments (McDonald et al. 1990, Patil 2002). In essence, it postulates that the total usage of each unit of a particular habitat is proportional to its suitability $\left[\left(u_{\mathbf{x}} / f_{\mathbf{x}}\right) \propto w_{\mathbf{x}}\right]$. Importantly, the apparent suitability (preference or avoidance) of habitat type $\mathbf{x}$ is conditional on the availability of all habitats in the environment (Johnson 1980) subject to any accessibility constraints (Matthiopoulos 2003). Apparent suitability can be modeled as any nonnegative-valued function of a linear predictor based on a vector of location-specific values for environmental covariates. The exponential function is, by far, the most appropriate from a theoretical perspective (McDonald et al. 1990, Manly et al. 2002, but see also Lele et al. 2013)

$$
w_{\mathbf{x}}=\exp \left(G_{\mathbf{x}}\right)
$$

where

$$
G_{\mathbf{x}}=\sum_{k=1}^{K} \sum_{r=0}^{2} \gamma_{r, k} x_{k}^{r}
$$

Here, habitat suitability is quantified by the parameters $\gamma_{r, k}$. This formulation of suitability is a HSF (also known as a resource selection function; Boyce and McDonald 1999). The similarity between the formulations for local fitness ( $F_{\mathbf{x}}$ in Eq. 6) and the linear predictor of suitability ( $G_{\mathbf{x}}$ in Eq. 9 ) is not coincidental. We discuss their possible connections in $A$ note on the relationship between partial fitness and habitat suitability. 
Bringing together Eqs. 2, 7, and 8 (and dropping the dummy variable $\mathbf{y}$ from the integral in the denominator) yields a general expression for average fitness

$$
F(f, \mathbf{N})=\frac{\int_{E} F_{\mathrm{x}} \exp \left(G_{\mathrm{x}}\right) f_{\mathrm{x}} d \mathbf{x}}{\int_{E} \exp \left(G_{\mathrm{x}}\right) f_{\mathrm{x}} d \mathbf{x}} .
$$

\section{Parametric Formulations of Habitat Availability}

Making use of Eq. 10 requires us to have a quantitative description of the environment's makeup. For any given environment and for predetermined cell sizes in $E$ - and $G$ spaces, it is possible to calculate the observed relative frequencies $\left(f_{\mathbf{x}}\right)$ of occurrence of each habitat as the proportion of the study area occupied by that habitat. The integrals in Eq. 10 could then be approximated by sums and calculated numerically. However, this can prove computationally expensive for the purposes of statistical estimation, particularly when $E$-space is high dimensional. For this reason, we pursue an analytical approach, capturing the complicated shape of habitat availability in $E$-space by its salient statistical properties. This can be done by parametrically approximating the empirical distribution of habitat availability in $E$-space by a Gaussian mixture. Since the values $f_{\mathbf{x}}$ in $E$-space are probability densities, the required approximating function must satisfy nonnegativity and unit-sum requirements. We use an $L$-mixture of $K$-variate Gaussian kernels (where $L$ is the number of kernels used in the mixture and $K$ is the dimensionality of $E$-space). There are several options regarding the kernels chosen to do this. For example, there are (1) Gaussian kernels including different variances in each environmental dimension and allowing for correlation between environmental variables, (2) different variances in each dimension but no correlation, and (3) fixed variance and no correlation. The trade-off here is between the flexibility of different kernels and the total number of kernels required to adequately approximate the shape of $f_{\mathbf{x}}$ within $E$-space. We selected option 2, because it offers the best compromise between biological realism and mathematical tractability. In addition, we elected to use the same set of $K$ variance parameters for each of the $L$ kernels. This gives the following approximation of habitat availability:

$$
\begin{aligned}
f_{\mathbf{x}} & =\sum_{l=1}^{L} \psi_{l} f_{l, \mathbf{x}} \\
& =\frac{1}{(2 \pi)^{\frac{K}{2}} \prod_{k=1}^{K} \sigma_{k}} \sum_{l=1}^{L} \psi_{l} \exp \left(-\frac{1}{2} \sum_{k=1}^{K}\left(\frac{x_{k}-\mu_{l, k}}{\sigma_{k}}\right)^{2}\right)
\end{aligned}
$$

where $f_{l, \mathbf{x}}$ is the probability density function (PDF) of the individual (lth) kernel, $\psi_{l}$ is the weight associated with the lth kernel, $\mu_{l, k}$ is the position of the lth kernel along the $k$ th environmental dimension, and $\sigma_{k}^{2}$ is the variance associated with the $k$ th environmental dimension. Since the kernel weights are positive and add up to 1, Eq. 11 satisfies both conditions for a PDF

$$
f_{\mathbf{x}} \geq 0, \quad \int_{E} f_{\mathbf{x}} d \mathbf{x}=1 .
$$

There are well-documented software tools for performing this decomposition that can work fast and robustly for high-dimensional $E$-spaces. We used the R package mclust (Fraley and Raftery 2002, Fraley et al. 2012). We provide further details of its usage in Parameter estimation from space-use and population time-series data, and a graphical example of its output is shown in Fig. 3.

\section{Connecting Habitat Use to Exponential Population GROWTH}

In the absence of density dependence, the population model in Eq. 1 becomes

$$
\frac{N_{t+1}}{N_{t}}=\exp (F(\mathbf{f})) .
$$

In this section, we obtain the intrinsic population growth rate $(r)$ on the right-hand side of this equation as a function of habitat suitability and habitat availability parameters. We focus on manipulating the expression for the average fitness $F(\mathbf{f})$. Incorporating Eqs. 6 and 9 into Eq. 10 gives the expression

$$
F=\frac{\int_{E}\left(\sum_{k=1}^{K} \sum_{r=0}^{2} \beta_{r, k} x_{k}^{r}\right) \exp \left(\sum_{k=1}^{K} \sum_{r=0}^{2} \gamma_{r, k} x_{k}^{r}\right) f_{\mathbf{x}} d \mathbf{x}}{\int_{E} \exp \left(\sum_{k=1}^{K} \sum_{r=0}^{2} \gamma_{r, k} x_{k}^{r}\right) f_{\mathbf{x}} d \mathbf{x}} .
$$

In Appendix A, with the aid of Eq. 11 we convert this into an integral-free expression. The population model implied by Eq. 1 with this density-independent definition of fitness is

$$
\frac{N_{t+1}}{N_{t}}=\exp \left(\frac{F_{1}}{F_{2}}\right)
$$

where $F_{1}, F_{2}$ are laid out in Appendix A: Eq. A.23 as functions of the coefficients describing habitat availability (the parameters $\mu_{l, k}, \sigma_{k}^{2}$ of the Gaussian approximation), habitat use (as quantified by the parameters $\gamma_{r, j}$ ) and habitat-related fitness (as quantified by the parameters $\beta_{r, j}$ ). The fact that these expressions are integralfree, and therefore computationally cheap, is particularly important for use in multidimensional statistical inference (see Parameter estimation from space-use and population time-series data).

\section{Connecting Habitat Use to Density-Dependent Population Growth}

Conspecific interference affects the population in two ways. First, the fitness that can be obtained from a crowded habitat is reduced, ultimately leading to a 
a) Food

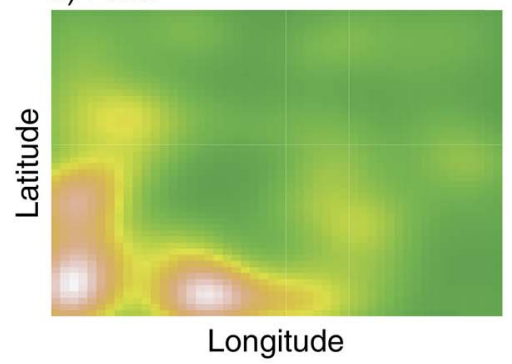

c) Fitness

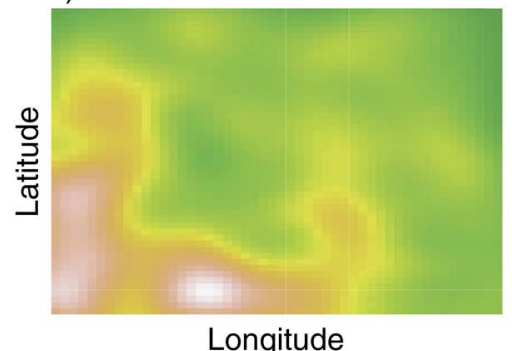

e) Numerical availability

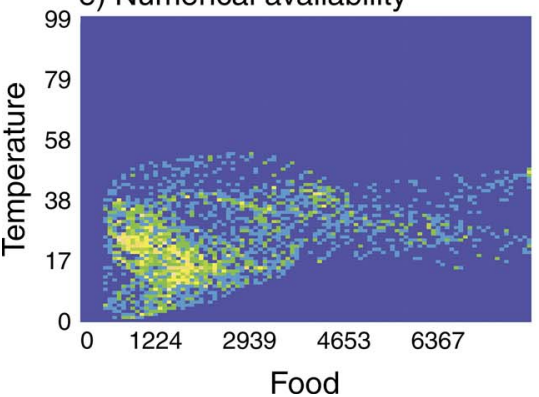

b) Temperature

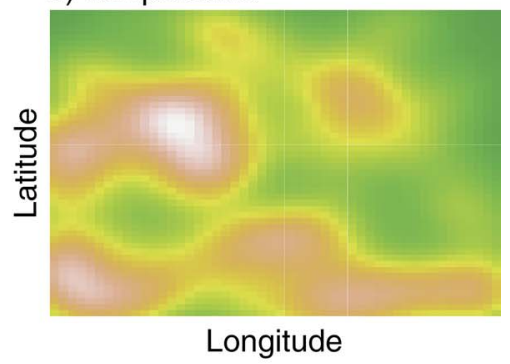

d) Usage

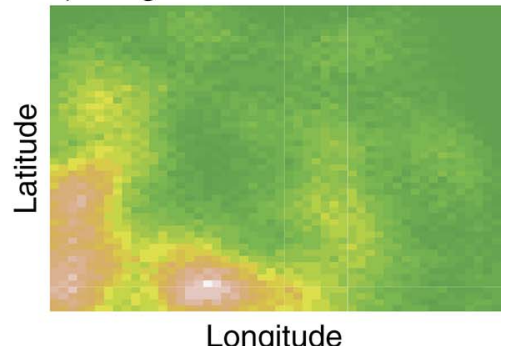

f) Approximated availability

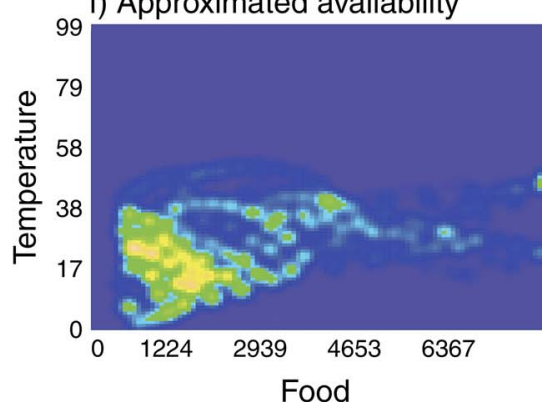

FIG. 3. In this example, two environmental variables, (a) the resource (food) and (b) the condition (temperature) combine to create different habitat types in different locations in geographical space ( $G$-space; latitude and longitude here are names for the axes of the simulation area). In this study, (c) the partial fitness of each location is determined by the local habitat. Through annual redistribution and differential population growth, this gives rise to (d) the usage distribution (here, shown when the population size has reached carrying capacity for this hypothetical landscape). We used a green-to-white color scale (terrain.colors() function in R; $\mathrm{R}$ Core Team 2014) to represent low-to-high values of each environmental variable in $G$-space. The availability of different habitats in environmental space ( $E$-space) can be visualized by counting the frequencies of occurrence of different habitats and using them (e) to generate a density plot. These numerical availabilities can be approximated by (f) a mixture of Gaussian kernels; in (e) and (f), the values listed for temperature and food are in arbitrary simulation units. In this example, the approximation comprises 300 kernels whose positions, variances, and associated weights were estimated by the R package mclust (Fraley and Raftery 2002, Fraley et al. 2012). We used a blue-to-yellow color scale (topo.colors() function in R) to represent low-to-high habitat availabilities in $G$-space.

reduction in population growth rates. This can be modeled by incorporating population density as a covariate to fitness, thus accounting for the extent of conspecific interference by treating it as a characteristic of an organism's local environment. The expected number of organisms using a single unit of area belonging to habitat $\mathbf{x}$ is

$$
\frac{u_{\mathbf{x}} N_{t}}{A f_{\mathbf{x}}}
$$

where $A$ is the total study area. Habitat usage in this expression is divided by $A f_{\mathbf{x}}$ because organisms experience crowding in $G$-space (i.e., units of area). If the unitary interference (defined as a proportional reduction in fitness; see assumption 5) caused to an individual by conspecifics is $b$, then the (reduced) partial fitness at that habitat is redefined as

$$
\tilde{F}_{\mathbf{x}}=F_{\mathbf{x}}-b \frac{u_{\mathbf{x}} N_{t}}{A f_{\mathbf{x}}} .
$$

The second effect of population density is on the resulting spatial distribution of mobile organisms. If individuals can avoid competition through relocating, they may increasingly be observed using suboptimal habitats that offer better prospects than the crowded (originally high-fitness) habitats. This will ultimately 
lead to a more homogeneous equilibrium distribution, approximating the ideal free distribution under high densities and leading to estimated habitat suitability coefficients $\gamma$ that vary as functions of habitat availability and population density. This effect is often ignored in the species distribution literature, but its existence underlines the need for distinguishing between habitat suitability coefficients (here, $\gamma$ ) estimated by species distribution models and the underlying coefficients of fitness (here, $\beta$ ). There are two ways to model this distinction. We may try to derive a theoretical relationship between the two types of coefficients from biological first principles. We begin the process in this study ( $A$ note on the relationship between partial fitness and habitat suitability). Alternatively, we may derive the values of the $\gamma$ coefficients directly from spatial data, using a habitat selection model for each new scenario of habitat availability and population density. This is the approach we have adopted for inference, in Parameter estimation from space-use and population time-series data.

The implications of density dependence for average fitness can be described by the following modification of Eq. 2:

$$
F\left(\mathbf{f}, N_{t}\right)=\int_{E} \tilde{F}_{\mathbf{x}} u_{\mathbf{x}} d \mathbf{x}
$$

where the expression $\tilde{F}_{\mathbf{x}}$ depends on population density (Eq. 17). By substituting Eqs. 7 and 17 into Eq. 18, we get

$$
F\left(\mathbf{f}, N_{t}\right)=\frac{\int_{E} F_{\mathbf{x}} w_{\mathbf{x}} f_{\mathbf{x}} d \mathbf{x}}{\int_{E} w_{\mathbf{x}} f_{\mathbf{x}} d \mathbf{x}}-b N_{t} A^{-1} \frac{\int_{E} w_{\mathbf{x}}^{2} f_{\mathbf{x}} d \mathbf{x}}{\left(\int_{E} w_{\mathbf{x}} f_{\mathbf{x}} d \mathbf{x}\right)^{2}} .
$$

The integrals in the density-dependent term of Eq. 19 have a direct geographical interpretation as the first and second moments of suitability

$$
E\left(w_{\mathbf{x}}\right)=\int_{E} w_{\mathbf{x}} f_{\mathbf{x}} d \mathbf{x}, \quad E\left(w_{\mathbf{x}}^{2}\right)=\int_{E} w_{\mathbf{x}}^{2} f_{\mathbf{x}} d \mathbf{x} .
$$

Therefore, the density-dependent term in Eq. 18 takes the form

$$
\begin{aligned}
-b N_{t} A^{-1} \frac{E\left(w_{\mathbf{x}}^{2}\right)}{E\left(w_{\mathbf{x}}\right)^{2}} & =-b N_{t} A^{-1} \frac{\operatorname{Var}\left(w_{\mathbf{x}}\right)+E\left(w_{\mathbf{x}}\right)^{2}}{E\left(w_{\mathbf{x}}\right)^{2}} \\
& =-b N_{t} A^{-1}\left(\mathrm{CV}\left(w_{\mathbf{x}}\right)^{2}+1\right)
\end{aligned}
$$

where $\mathrm{CV}\left(w_{\mathbf{x}}\right)$ is the coefficient of variation of habitat suitability across the environment. A completely uniform landscape comprising solely of habitat $\mathbf{x}$ would be characterized by $\mathrm{CV}\left(w_{\mathbf{x}}\right)=0$, which yields the nonspatial version of density dependence $-b N_{t}$. In this situation, the population's dynamics are described by

$$
N_{t+1}=N_{t} \exp \left(F_{\mathbf{x}}-b N_{t}\right)
$$

which is the well-known Ricker model (e.g., Matthiopoulos 2011:132)

$$
N_{t+1}=N_{t} \exp \left(r_{\max }\left(1-\frac{N_{t}}{N^{*}}\right)\right) .
$$

This correspondence implies that the intrinsic growth rate of a population living in a uniform environment is $r_{\max }=F_{\mathbf{x}}$ and the interference parameter has the alternative definition $b=r_{\max } / N^{*}$, expressed in terms of the population's intrinsic growth rate $\left(r_{\max }\right)$ and carrying capacity $\left(N^{*}\right)$.

Eq. 21 suggests that, for populations of the same size, the impact of density dependence will be greatest for organisms that perceive their environment as more heterogeneous in quality, i.e., when there are hotspots of extremely good habitat, the growth of the population will be more prone to displaying the effects of crowding.

Using the results from Appendix B, with the specific formulations for availability (Eq. 11), and fitness (Eq. 6), we obtain an integral-free expression for Eq. 19. The population model implied by Eq. 1 with this densitydependent definition of fitness is

$$
\frac{N_{t+1}}{N_{t}}=\exp \left(\frac{F_{1}}{F_{2}}-b N_{t} A^{-1} g \frac{F_{3}}{F_{2}^{2}}\right)
$$

where the expressions $F_{1}, F_{2}$ are the same as in Eq. 15 and, along with the new expression $F_{3}$, are written as functions of the coefficients describing habitat availability (the parameters $\mu_{1, k}, \sigma_{k}^{2}$ of the Gaussian approximation), habitat use (as quantified by the parameters $\gamma_{r, j}$ ) and habitat-related fitness (as quantified by the parameters $\beta_{r, j}$ ). The expanded versions of $F_{1}, F_{2}, F_{3}$ can be found in Appendix B: Eq. B.7.

\section{A Note on the Relationship Between Partial Fitness and Habitat Suitability}

Organisms will tend to be found in habitats that benefit their fitness, but the efficiency with which they do this will vary. In general, it should be possible to devise a mathematical relationship between a habitat's partial fitness and its apparent suitability. In our framework, we have tried to facilitate this task by using identical polynomial formulations for the linear predictors of these two quantities

$$
F_{\mathrm{x}}=\sum_{k=1}^{K} \sum_{r=0}^{2} \beta_{r, k} x_{k}^{r} \quad G_{x}=\sum_{k=1}^{K} \sum_{r=0}^{2} \gamma_{r, k} x_{k}^{r} .
$$

However, the fitness that can potentially be afforded by unoccupied habitat will be constant, and therefore the coefficients of fitness (the betas) are a characteristic of the species responding to this habitat. In contrast, the coefficients of apparent suitability (the gammas) will vary as functions of habitat availability and population density. This is the important distinction that is missing from methods such as environmental niche factor analysis 
(Hirzel et al. 2002) and related multivariate methods, also known as "profile" methods (McDonald et al. 2013). The dependence of the $\gamma$ 's on habitat availability has been previously modeled (Matthiopoulos et al. 2011), so we focus here on the dependence of apparent habitat suitability on prevailing population density.

The relationship between partial fitness and habitat usage is likely to be nontrivial (Van Horne 1983). The ability of organisms to perceive and respond to spatial gradients of fitness depends on their sensory and movement capabilities. Highly perceptive and mobile organisms will be able to discover global optima in habitat suitability. However (due to differential mortality resulting from spatial variations in fitness), even organisms with no perception (e.g., random walkers) will present spatial patterns that, to a much smaller extent, track fitness gradients. The ideal approach to unraveling the relationship between partial fitness and habitat usage is to derive spatial redistribution models from first principles pertaining to individual behavior and movement. These mechanistic models need to include candidate rules about how individuals respond to their environment, their conspecifics, and their own past experience. Despite their high demands for mechanical detail, such models exist, and their embedded parameters have, in certain cases (e.g., Moorcroft and Lewis 2006), been estimated from spatial data. However, our approach offers a shortcut to the problem. Mathematically, the simplest relationship between fitness and habitat suitability can be achieved by linking them via a proportionality relationship

$$
\gamma_{r, k}=h_{k}(N) \beta_{r, k}
$$

where the function $h_{k}$ scales the fitness coefficient of the $k$ th environmental covariate (hence, it applies to both the first- and second-order coefficients of quadratic responses). We will argue here that even such a simple formulation can carry many biologically realistic properties. Placing Eq. 26 into the combination of Eqs. 8 and 9 gives

$$
w_{\mathbf{x}}=\prod_{k=1}^{K} \exp \left(h_{k}(N) \sum_{r=0}^{2} \beta_{r, k} x_{k}^{r}\right) .
$$

For a given value of density $N$, the value of $h$ is determined by the behavioral characteristics of the species, particularly its ability to track improvements in fitness along environmental gradients. It therefore quantifies how close the organism is to the classic ideal of optimal resource use. Large values of the $h$ 's correspond to highly responsive organisms free of competition. These animals should be expected to spend most of their time in the highest-fitness habitat, even if it is insufficient. Indeed, combining perceptive animals living in low-density environments (i.e., very high values of $\left.h_{k}(N)\right)$ with a habitat $\mathbf{x}$ whose partial fitness is particularly high compared to all others (i.e., comparatively high values for all $\sum_{r=0}^{2} \beta_{r, k} x_{k}^{r}$ ), yields high values of apparent suitability (according to Eq. 27). In the limit, for such high values, the normalization in Eq. 7 gives

$$
\lim _{w_{x} \rightarrow \infty} u_{\mathrm{x}}=1 \text {. }
$$

This implies that populations of highly perceptive and mobile organisms at low densities will be observed to use this particularly good habitat, almost to the exclusion of all others. Eq. 28 will be more easily satisfied if the concavity of the exponential transformation is high, when the large gamma coefficients produced by Eq. 26 will present a sharp drop from complete occupancy of optimal habitats to near-zero usage of (even slightly) suboptimal habitats (while the underlying fitness values of these habitats, described by the beta coefficients, remain unchanged). However, real animals are never omniscient and only rarely free of competition. Hence, most real situations of near-optimal usage can be captured with sufficiently large, positive values of $h$.

Negative values of $h$ correspond to maladaptive habitat selection (the organisms actively pursuing lower-fitness habitat types). If the population is studied at a biologically unrealistic spatial scale (i.e., larger than their movement or perception range), models fitted to such data can give the appearance of maladaptive behavior (Beyer et al. 2010).

When population density is high, we would expect the population to deviate from just using optimal habitats (i.e., the values of $h$ should decrease from their values at low $N$ ). In general, we can interpret $\exp \left(F_{\mathbf{x}}\right)$, the expected rate of population growth corresponding to a particular habitat $\mathbf{x}$, as a measure of the quality of that habitat. As the function $h(N)$ approaches 1, the estimated value of the habitat selection function for any habitat $\mathbf{x}$ becomes equal to the intrinsic growth rate of a population living exclusively in such a habitat (i.e., $\exp \left(G_{\mathbf{x}}\right)=\exp \left(F_{\mathbf{x}}\right)$ ). In other words, a quantity proportional to the expected usage of a single unit of space of this habitat $\left(\exp \left(G_{\mathbf{x}}\right)\right.$; see Linking fitness, habitat suitability, and habitat use) becomes equal to the intrinsic quality of that habitat $\left(\exp \left(F_{\mathbf{x}}\right)\right)$. Hence, in the presence of intraspecific competition, the special case $h=1$ gives rise to an analogue of the ideal free distribution (Fretwell and Lucas 1970). The case $h=0$ gives rise to unselective use of space, irrespective of the fitness consequences of different habitats, corresponding to uniformly distributed populations (in expectation).

Given these cornerstone scenarios, there are several possibilities for specifying $h$ as a function of population size. If the data on space use have been collected within a biologically realistic spatial scale (e.g., territories of individual animals), then the function could be set to satisfy the properties

$$
\frac{d h}{d N}<0, h(N)>0 .
$$

The first property expresses the notion that as population density increases, suboptimal habitats are 
used more frequently. Since this property can, alternatively, be thought of as a reduction in the efficiency with which organisms congregate at the highest-fitness habitats, we emulate the behavior by specifying $h$ as a decreasing function of $N$. The second property prevents high population density from inverting the ranking of habitats according to fitness (in this way, based on observed habitat usage, a high-fitness habitat will always appear to be preferred at least as much as a low-fitness habitat). The following expression is an example satisfying these conditions

$$
\begin{aligned}
h_{k}(N)= & c_{0, k}+\left(c_{1, k}-c_{0, k}\right) \frac{c_{2, k}}{c_{2, k}+N} \\
& \left(c_{1, k}>c_{0, k}, \quad c_{0, k}, c_{1, k}, c_{2, k}>0\right) .
\end{aligned}
$$

This describes a population that, at low densities, aggregates tightly around the highest fitness habitats $\left(h_{k}(0)=c_{1, k}\right)$. At higher densities, the population also uses suboptimal habitats because $\lim _{N \rightarrow \infty} h_{k}(N)=c_{0, k}$. The rate at which this lower value is approached, is determined by $c_{2, k}$. Setting $c_{0, k}=1$ generates an ideal free distribution at high densities $\left(\lim _{N \rightarrow \infty} h(N)=1\right)$.

An important distinction between the material presented in this section and the previous (on density dependence) is that expressions such as Eq. 30 can capture the efficiency with which organisms respond to underlying reductions in fitness brought about by the density-dependent effects described in Connecting habitat use to density-dependent population growth. Estimating the parameters of functions such as Eq. 30 could therefore enable us to model the mismatches between the observed changes in space use and the underlying changes in realized fitness. Although the estimation methodology for these parameters falls outside the remit of the present study, future work in this direction could fruitfully investigate the quantitative and empirical connections between the efficiency with which apparent habitat suitability tracks fitness and how this relationship varies along different environmental variables, in different environmental makeups, and under variable population densities. In the following section, we bypass these issues by assuming no connection between the gammas and the betas, and rather estimating both of those sets of parameters independently from the data.

\section{Parameter Estimation from Space-Use and Population Time-Series Data}

Our statistical inference on environmental, space-use, and population data follows three corresponding stages that, in this first incarnation of the methodology, are performed in a sequential (rather than integrated) fashion. We take this approach so as to more straightforwardly illustrate our model's fitting by use of broadly understood tools (generalized linear models) in the software language R (R Core Team 2014).

\section{Stage 1: approximation of habitat availabilities in E-space.}

The objective of this first stage is to approximate the frequency with which different habitats occur in $E$-space by a mixture of multivariate Gaussians (see Eq. 11), each with the same variance-covariance (var-cov) matrix (here, we set the covariances to zero and used different variances for each environmental dimension). We performed this task using the R package mclust (Fraley and Raftery 2002, Fraley et al. 2012). The requisite input for the command mclust is a data frame with rows representing points in $G$-space and columns representing environmental variables. The package can perform model selection using the Bayes information criterion to settle on the number of mixture components and the var-cov structure of each Gaussian component. However, since we are interested in using the clustering as an accurate approximation of the data, rather than as a parsimonious description of some underlying truth, we fixed the number of kernels to a high number $(L=300)$; note that in the $\mathrm{R}$ code in Supplement 1, this often results in a warning message because for some of the less complicated $E$-spaces, not all of these kernels are needed to achieve a good approximation, so not all of these kernels can be assigned by mclust(). The function's output (in addition to the position of each component and its var-cov structure) includes the mixture proportions (the $\psi$ 's of Eq. 11) of the components. The simple var-cov structure stipulated by our approach corresponded to the predefined model "EEI" in mclust. An example of how a realization of our two-dimensional $E$ space is approximated by mclust is shown in Fig. 3.

\section{Stage 2: fitting habitat-selection functions to annual snapshots of usage data}

For any given snapshot of spatial data, the apparent habitat suitability coefficients $\gamma$ of the HSF can be estimated using GLMs. However, as discussed in A note on the relationship between partial fitness and habitat suitability, these coefficients are conditional on the environment (i.e., the complete profile of habitat availabilities $\mathbf{f}$ ) and the population densities in which the model-fitting data were collected. It is well-known that HSF coefficients are sensitive to changes in habitat availability (Randin et al. 2006, Beyer et al. 2010, McLoughlin et al. 2010, Matthiopoulos et al. 2011). This effect, known as a functional response to habitat availability (Arthur et al. 1996, Mysterud and Ims 1998, Mauritzen et al. 2003, Matthiopoulos et al. 2011, Moreau et al. 2012, Aarts et al. 2013) is particularly important for our study because we wish to derive inferences for multiple environments under different population sizes. There are three statistical approaches for dealing with variability in the HSF coefficients: (1) Post hoc estimation, in which the HSF is fit separately to each environment and the joint HSF parameters are derived as summaries from the distribution of parameter estimates under all scenarios (Moreau et al. 2012). (2) 
Mixed-effects estimation, in which the deviations of HSF parameters from their fixed-effects value are modeled as a random effect specific to each environment (Gillies et al. 2006, Duchesne et al. 2010, Gillies and St Clair 2010). (3) Generalized functional responses (GFR); by expressing the HSF coefficients as flexible functions of habitat availability, it can be shown that the resulting statistical model has a simple structure containing statistical summaries of availability (e.g., mean values of resources for each environment) and their interactions with the local values of environmental variables (Matthiopoulos et al. 2011, Aarts et al. 2013).

In general, we favor the third approach because it estimates the parameters of the functional response to habitat availability (unlike approach 2), and does so in a single inferential step (unlike approach 1), hence offering greater predictive potential for unobserved environments and a more integrated treatment of uncertainty. We therefore used a GFR to calculate scenario-specific coefficients (see Supplement 1: Part 3). Since HSF coefficients are also sensitive to population density, the current size of the population was also included in the GFR.

\section{Stage 3: estimation of population dynamics parameters}

Taking the more general case of a density-dependent population in Eq. 24, the previous two stages provide estimates for the parameters $\mu_{l, k}, \sigma_{k}^{2}$ of the Gaussian approximation and $\gamma_{r, j}$ for habitat suitability. The parameters that now need to be evaluated are the intercept $\beta_{0}=\sum \beta_{0, k}$ that relates to baseline population growth, the fitness coefficients $\beta_{r, j}$, and the coefficient $b$ describing attrition due to density dependence. The model in Eq. 24 can be rewritten with the details provided in Appendix $\mathrm{B}$ to arrange these missing coefficients into a single linear predictor

$$
\begin{aligned}
\frac{N_{t+1}}{N_{t}} & =\exp \left(\beta_{0}+\left\{\sum_{k=1}^{K} \beta_{1, k} \phi_{1, k}+\beta_{2, k} \phi_{2, k}\right\}-b \phi_{3} N_{t}\right) \\
\phi_{1, k} & =\frac{1}{F_{2}} \sum_{l=1}^{L}\left[\psi_{l} \Theta_{l} \frac{\left(\gamma_{1, k} \sigma_{k}^{2}+\mu_{l, k}\right)}{\left(1-2 \gamma_{2, k} \sigma_{k}^{2}\right)}\right] \\
\phi_{2, k} & =\frac{1}{F_{2}} \sum_{l=1}^{L}\left[\psi_{l} \Theta_{l} \frac{\sigma_{k}^{2}}{\left(1-2 \gamma_{2, k} \sigma_{k}^{2}\right)}\left(1+\frac{\left(\gamma_{1, k} \sigma_{k}^{2}+\mu_{l, k}\right)^{2}}{\sigma_{k}^{2}\left(1-2 \gamma_{2, k} \sigma_{k}^{2}\right)}\right)\right] \\
\phi_{3} & =N_{t} g \frac{F_{3}}{F_{2}^{2}}
\end{aligned}
$$

in which $F_{1}, F_{2}, F_{3}, \Theta_{l}$, and $g$ are as defined in Appendix B: Eq. B.7 $\left(\Theta_{l}\right.$ and $g$ are notational devices). Note therefore that the first line of this model comprises a linear predictor involving the new, constructed covariates $\phi_{1, k}, \phi_{2, k}, \phi_{3}$ and the coefficients $\beta_{0}, \beta_{1, k}, \beta_{2, k}, b$ for $k=1, \ldots, K$. Therefore, for $K$-dimensional $E$-space, this model will be required to estimate up to $2(K+1)$ coefficients (fewer if non-quadratic forms are needed for some of the environmental variables). Hence, we will require at least as many instances of data. Each data instance must be represented by (1) environmental data corresponding to the observed usage (for stages 1 and 2), (2) a sample of spatial usage data (for stage 2), and (3) a measurement of population growth, or two successive measurements of population size (for stage 3). For example, in the case of a population using two resources (one coefficient each), whose distribution is driven by two conditions (two coefficients each), we would expect to need a minimum sample size of eight data instances (but ideally, closer to twice that number). The model in Eq. 31 can be estimated as the following log-linear GLM:

$$
\begin{aligned}
& N_{t+1} \sim \operatorname{Poisson}\left(\lambda_{t}\right) \\
& \lambda_{t}=\exp \left(\beta_{0}+\beta_{1,1} \phi_{1,1}+\cdots+b \phi_{3, t}+\log \left(N_{t}\right)\right) .
\end{aligned}
$$

Use of Poisson stochasticity is purely illustrative. Multiple sources of uncertainty may lead to overdispersed population data, such as demographic stochasticity, error in the estimates of population size, stochastic perturbations affecting the population but not its environment, or stochasticity in environmental variables not available for inclusion in the HSF. In such cases, it may be preferable to adopt a quasiPoisson, gamma, negative-binomial, or log-normal stochastic component, as provisioned by statistical packages, such as $\mathrm{R}$.

For comparison purposes, a nonspatial version of the population model in Eq. 32 can be obtained by regressing future population size against the average values of environmental variables, using both first- and second-order terms to capture non-monotonic responses to conditions. Henceforth, we refer to this as the meanfield model

$$
\begin{aligned}
N_{t+1} & \sim \operatorname{Poisson}\left(\lambda_{t}\right) \\
\lambda_{t}= & \exp \left(\delta_{0}+\delta_{1,1} \bar{X}_{1}+\delta_{1,2} \bar{X}_{1}^{2}+\cdots+\delta_{K, 1} \bar{X}_{K}+\delta_{K, 2} \bar{X}_{K}^{2}\right. \\
& \left.+\delta_{N} N_{t}+\log \left(N_{t}\right)\right)
\end{aligned}
$$

where $\delta$ is a regression coefficient to be estimated by fitting the mean-field model to population data $\left(N_{t+1}\right.$, $\left.N_{t}\right)$ and environmental averages $\left(\bar{X}_{1}, \ldots, \bar{X}_{K}\right)$.

Arguably, this staged approach can have two disadvantages. First, if only the point estimates of parameters from each stage are used for the calculations in the next stage, then uncertainty is not propagated to the final output. We addressed this by resampling from the full variance-covariance structure of intermediate models to perform the calculations in the following stages (an approach equivalent to a parametric bootstrap). The second drawback with our staged approach is that data at later stages do not have an opportunity to inform the likelihood of fits at earlier stages. Hence, it is conceivable that data on population change might (if allowed) be able to have some bearing on the parameter estimates for habitat suitability. These potential gains in inferential 
power can only be achieved with an integrated fitting approach, which may be part of future work.

\section{Simulation}

The simulation aimed to adhere to our Ecological scope and simplifying assumptions and to include the following features: (1) spatially autocorrelated environments, (2) habitat-influenced individual movement, and (3) habitat-related population growth. The full code for the simulation and the associated analysis can be found in Supplement 1: Part 1.

We generated synthetic environments on gridded, 50 $\times 50 \mathrm{G}$-spaces comprising one resource (e.g., food) and one condition (e.g., temperature). A geographical map for each of these two variables was generated independently of each other using a seeding-and-smoothing method (listed as function environ() in Supplement 1). During the seeding process, a random number of locations were selected in space, according to a planar gradient of randomly selected slope. During the smoothing process, a Gaussian kernel of a random bandwidth was placed on each of the seed locations. The superposition of these Gaussian kernels created the environmental layer, which was then normalized to a randomly selected mean value for that environmental layer. Note that this use of Gaussian kernels simply aims to create autocorrelated environmental layers in $G$ space, so it is different from the Gaussian decomposition of the resulting habitat availabilities in E-space (described in Parametric formulations of habitat availability). In particular, the usability and efficiency of the Gaussian decomposition in $E$-space does not rely on the $G$-space data coming from a Gaussian field.

Each simulation year was subdivided into 12 "months." In each month, an animal would gain/lose condition, relocate in the landscape, and potentially die. We specified the profitability of any given cell in the landscape as an incremental change in each animal's current condition. The contribution to an animal's condition $\left(\Delta C_{\mathrm{s}}\right)$ offered by any point $\mathbf{s}$ in $G$-space was expressed as a polynomial expression in the local density of food $(R)$ and value of temperature $(T)$

$$
\Delta C_{\mathbf{s}}\left(R_{\mathbf{s}}, T_{\mathbf{s}}\right)=a_{0}+a_{1} R_{\mathbf{s}}+a_{2} T_{\mathbf{s}}+a_{3} T_{\mathbf{s}}^{2}
$$

where $a$ is a simulation parameter that quantifies the true contribution of food and temperature to the change in the condition of an animal per unit of time spent in habitat $\mathbf{x}$.

The coefficient values were selected so that the parabola in temperature was pointing downward $a_{3}<$ 0 and, in the absence of food, an individual's condition deteriorated (i.e., $\Delta C_{\mathbf{s}}\left(0, T_{\mathbf{s}}\right)<0$ implying, $a_{0}<a_{2}^{2} / 4 a_{3}$. Following an initial random placement of 10 individuals in month 1 of year 1 , animals were allowed to move in the landscape subject to toroidal boundaries (animals exited the landscape at one edge, reentered at the same position of the opposite edge). During movement, we updated the position of each individual as follows: the profitabilities of the combined set of the current cell with its von Neumann neighborhood were perturbed by an error term $\varepsilon_{\mathbf{s}} \sim N\left(0, \sigma^{2}\right)$ to represent imperfections in the organisms' perception of the profitability of each spatial cell. The cell with the maximum perceived value from this set, $\max \left(\Delta C_{\mathbf{s}}+\varepsilon_{\mathbf{s}}\right)$ was located and the individual was placed there. While the individual remained there, the profitability of that location was decreased by a fixed amount to represent conspecific attrition. No resource depletion was assumed. The process was repeated until all the individuals in the population had been given an opportunity to move. Note that the variance of the perception error was constant but the values of profitability could get continuously homogenized through the effect of attrition. Hence, this algorithm has the appealing property that, under conditions of crowding, current peaks in the spatial map of fitness become comparatively harder to detect. Mortality was implemented on a monthly basis as a Bernoulli process for each individual with a probability of survival dependent on current condition via a logit link. Fecundity was implemented on an annual basis as a Poisson process for each individual with a breeding rate depending log-linearly on current condition. Complete data on the population's spatial distribution and total population size $\left(N_{t}\right)$ were collected by the simulation just prior to breeding taking place. Simulations were initialized via a 20 -year burn-in period, during which populations were maintained to a low size of 100 individuals by the removal of surplus. This was done to ensure there were no artifacts in the early simulation years due to the fact that all founder individuals were initialized at a low energy level.

\section{Simulation Experiments}

We focused on our framework's descriptive and predictive ability under high and low data availability. Space-use and population data were produced for randomly generated environmental scenarios inhabited by founder populations (10 individuals) and, depending on environmental makeup, reaching sizes of thousands or tens of thousands over periods of 30 simulation years. We produced two data sets, one for fitting the model (20 environmental scenarios) and one for validating it (100 scenarios). The complete set of 120 population trajectories produced by these spatial simulations is shown in Fig. 4. For the high data availability experiments ( 1 and 2), models were fitted to the complete data set of 20 scenarios, each scenario containing a 30 -year population time series (i.e., a total of 600 sampling instances) and each year containing a $50 \times 50$ spatial layer of usage (i.e., a total of $1.5 \times 10^{6}$ spatial data points). For the low data availability experiments (3), we performed a systematic exploration of the method's performance, reporting robust results for values as low as $0.5 \%$ of the high data availability experiments (a total of 60 sampling instances and 7500 spatial observations). 


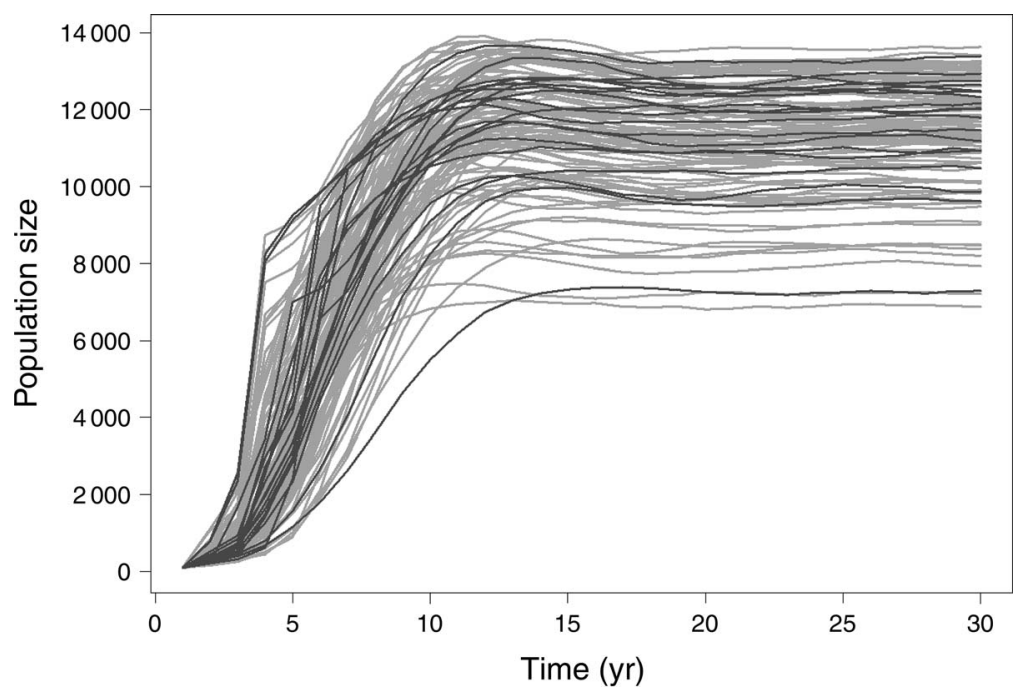

FIG. 4. Population trajectories generated by our individual-based population model over 30 simulated years. The simulations were spatially explicit, but this figure only shows the time series for total population size just prior to the breeding season. The dark gray trajectories were used for fitting the model to data (fitting data set). The light gray trajectories were used for the prediction experiments (validation data set).

Simulation experiment 1: descriptive ability of the model

In this experiment, we compared the ability of the spatial and mean-field models (Eqs. 32 and 33, respectively) to fit the data. We investigated goodness of fit to the observed population growth rates (Fig. $5 \mathrm{a}, \mathrm{b}$ ), as well as the observed carrying capacities (Fig. $6 a, b)$. The observed growth rates were calculated as $\log \left(N_{t+1}\right)-\log \left(N_{t}\right)$ and fitted growth rates were given from $\log \left(\hat{N}_{t}+1\right)-\log \left(\hat{N}_{t}\right)$, where $\hat{N}_{t+1}$ was the expected population size as estimated by Eqs. 32 and 33 for the spatial and mean-field models, respectively. Goodness of fit for the carrying capacity was depicted by plotting each of the last 20 years of observed population sizes from each of the 20 fitting simulations against the corresponding fitted sizes. Estimated parameter values were biologically consistent for both models. For example, fitness increased with food, and the densitydependent parameters always took negative values. Model AICs and the graphical comparisons in Figs. 5 and 6 indicated that the spatial model performed consistently better than the mean-field model.

\section{Simulation experiment 2: Predictive ability of the model}

For this experiment, we used the 100 validation environmental scenarios. We provided the spatial and mean-field models with the necessary environmental information for these new scenarios, but retained the model parameters (for both the HSF and population models) estimated from the original 20 scenarios. We compared the ability of the spatial and mean-field models to predict the growth rates and carrying capacities of a species in those 100 new environments. The annual growth rates were calculated as in experiment 1, and the predicted carrying capacities were calculated by setting $N_{t+1}=N_{t}$, in Eqs. 32 and 33. The estimators for the carrying capacities for the spatial and mean-field models respectively are

$$
\begin{aligned}
& \hat{N}^{*}=\frac{1}{b \phi_{3, t}}\left(\beta_{0}+\beta_{1,1} \phi_{1,1}+\cdots\right) \\
& \hat{N}^{*}=\frac{1}{\delta_{N}}\left(\delta_{0}+\delta_{1} \bar{X}_{1}+\cdots\right) .
\end{aligned}
$$

We found that the spatial model outperformed the mean-field predictions for both growth rates (Fig. 5c, d) and carrying capacities (Fig. 6c, d).

\section{Simulation experiment 3: Sensitivity of predictive ability to data availability}

The model-fitting data set comprised descriptions of different environmental scenarios, records of spatial usage, and time series of population size. To examine the sensitivity of the model's performance on these three types of information, data impoverishment was performed on each of them separately. We repeated the model-fitting procedure, each time incrementally reducing the fitting data, such that the new sample size $v$ was a decreasing proportion of the initial sample size $V$ (i.e., $v$ $=V, V / 2, V / 3, \ldots, V / 10)$. From each model fit on the impoverished data sets, we generated predictions of growth rate and carrying capacity for the 100 validation scenarios. Each annual prediction yielded a residual from the known, true value, and the 95th percentiles from the set of these residuals were used to summarize accuracy and precision for growth rate and carrying capacity separately. For each of these manipulations, we also traced the AIC of each model. The set of our results can be found in Appendix C. In summary, we found that 


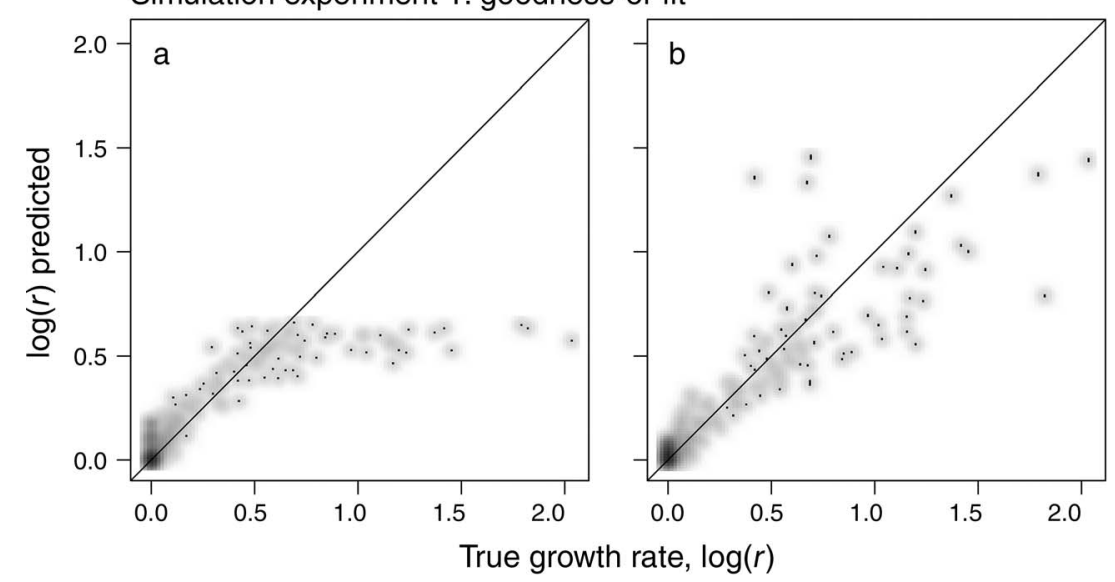

Simulation experiment 2: predictive ability

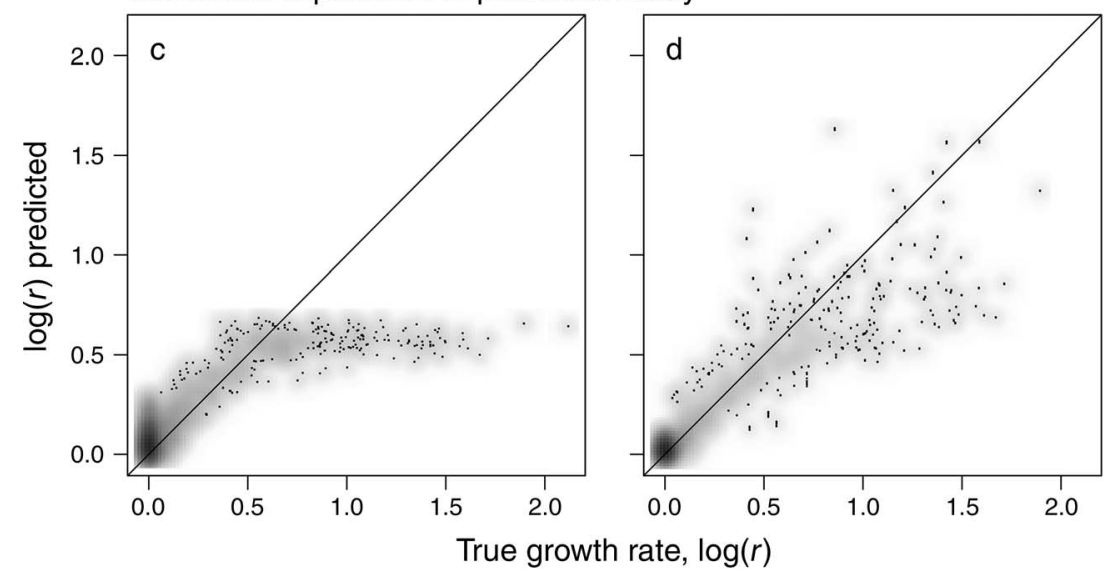

Simulation experiment 3: prediction with impoverished data

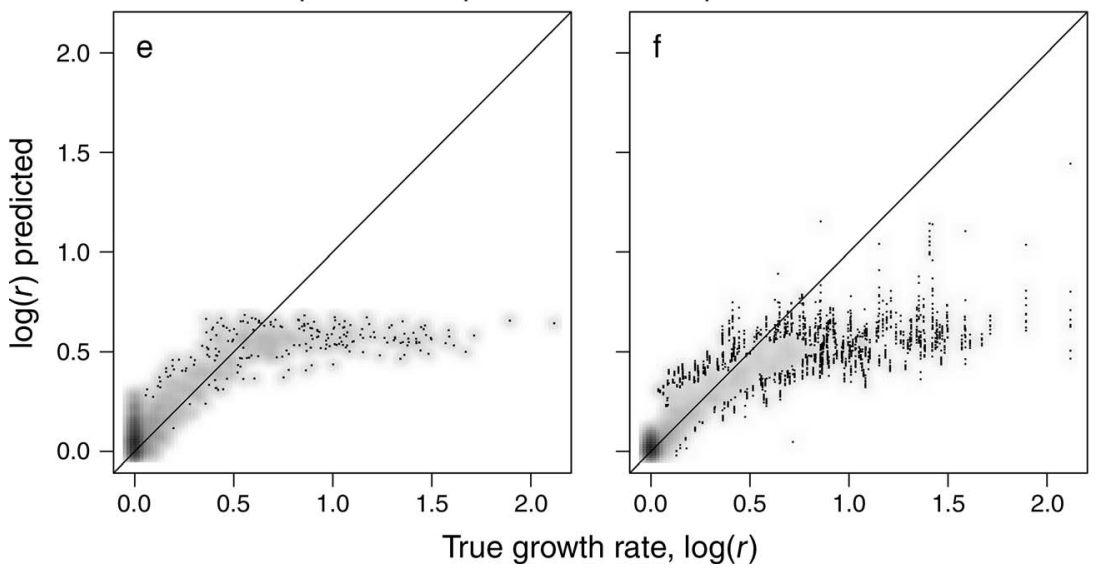

FIG. 5. Illustrative comparison for population growth rates $(r)$ between the mean-field (a, c, e) and spatial (b, d, f) models over three different simulation experiments. Experiment 1 ( $\mathrm{a}$ and $\mathrm{b}$ ), examined goodness of fit; the ability of the two models to capture the observed growth rates in the complete data set (comprising 20 environmental scenarios, each run for 30 years and recording 2500 spatial observations for each year). Experiment 2 ( $\mathrm{c}$ and d) examined predictive performance; the ability of the two models to anticipate population growth rates in previously unobserved landscapes. The models generating these predictions were also fitted to the complete data set. Experiment 3 (e and f) examined predictive performance under impoverished data sets. The predictions shown here are from models with less than $0.5 \%$ of the data used for the others (comprising three years per scenario and 125 spatial cells observed per year). To correctly represent the density of multiple overlapping data points, we have visualized these using the smoothScatter() function in R. This function plots a kernel-smoothed version of the data (shown as shades of gray) as well as a subset of the most outlying data as black points. Here, we plotted 50 outliers for the goodness-of-fit plots (a and b), 200 for the mean-field predictive plots (c and e), and 2000 outliers ( 200 predictions $\times 10$ bootstrap resamples for each prediction) for the spatial predictive plots ( $\mathrm{d}$ and $\mathrm{f})$. 
Simulation experiment 1: goodness-of-fit

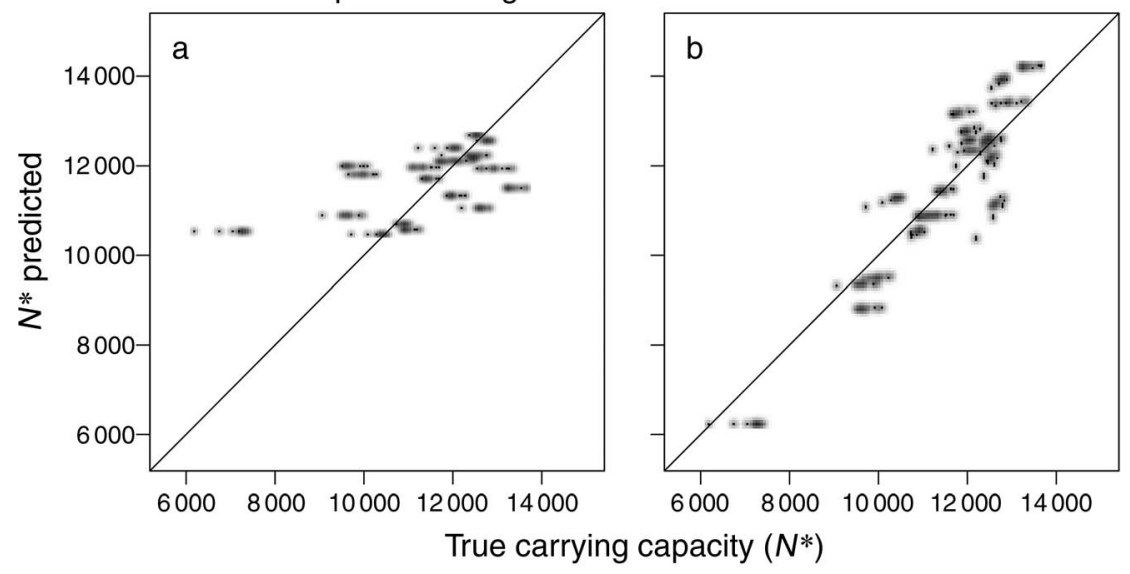

Simulation experiment 2: predictive ability

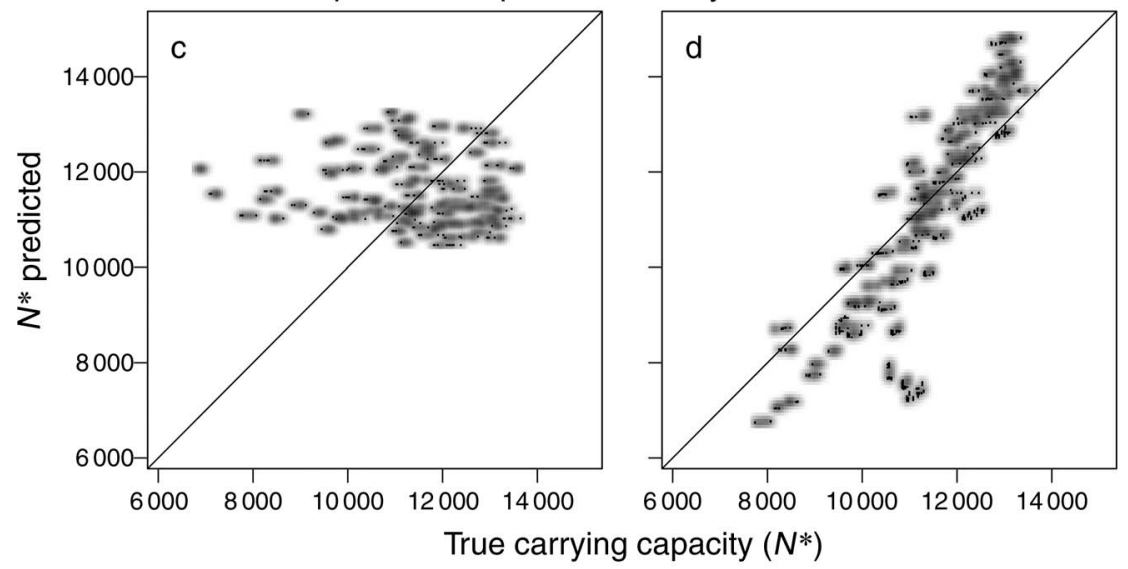

Simulation experiment 3: prediction with impoverished data

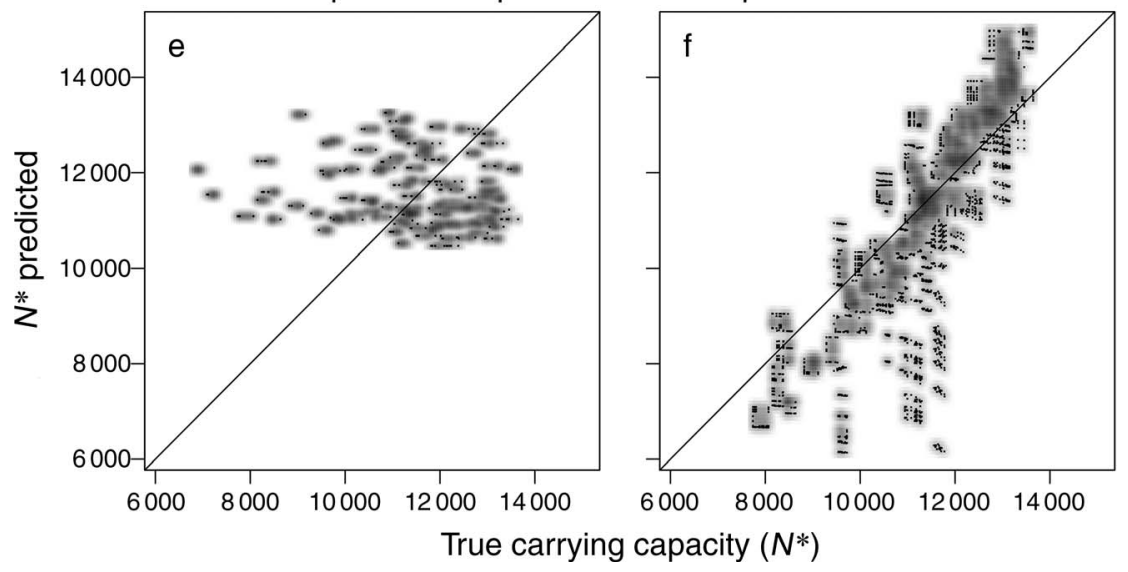

FIG. 6. Illustrative comparison for population carrying capacity $\left(N^{*}\right)$ between the mean-field (a, c, e) and spatial (b, d, f) models over three different simulation experiments. Experiment 1 ( $a$ and b) examined goodness of fit; the ability of the two models to capture the observed growth rates in the complete data set (comprising 20 environmental scenarios, each run for 30 years and recording 2500 spatial observations for each year). Experiment 2 ( $\mathrm{c}$ and d) examined predictive performance; the ability of the two models to anticipate the carrying capacities of previously unobserved landscapes. The models generating these predictions were also fitted to the complete data set. Experiment 3 (e and f) examined predictive performance under impoverished data sets. The predictions shown here are from models with less than $0.5 \%$ of the data used for the others (comprising three years per scenario and 125 spatial cells observed per year). To correctly represent the density of multiple overlapping data points, we have visualized these using the smoothScatter() function in R. This function plots a kernel-smoothed version of the data (shown as shades of gray) as well as a subset of the most outlying data as black points. Here, we plotted 50 outliers for the goodness-of-fit plots (a and b), 200 for the mean-field predictive plots (c and e) and 2000 outliers (200 predictions $\times 10$ bootstrap resamples for each prediction) for the spatial predictive plots (d and $\mathrm{f})$. 
our method's performance relied most crucially on having sufficient environmental and population contrast. Population contrast requires observations at saturated and pre-saturated densities (i.e., replication once at carrying capacity was less informative). Lack of environmental contrast implies that the method will not perform well if the species is observed in only a few distinct environmental scenarios, compared to the number of environmental covariates being considered. Sensitivity on the amount of spatial data was the lowest, as long as there was enough spatial data for an HSF to detect the spatial variations in distribution. Having explored sensitivity in this fashion, we specified one depauperate data set that lacked both population and spatial information. In particular, we only used three (first, middle, last) out of the maximum of 30 years from each of 20 fitting scenarios and we kept information on only 125 spatial cells out of a maximum of 2500 . This corresponds to a removal of $\sim 99.5 \%$ of the less-crucial information in the data. Although visibly impaired, the spatial model's ability to predict population growth remained higher than the mean-field model's (Fig. 5e, f). The spatial model's ability to predict carrying capacity remained relatively unaffected by the information loss (Fig. 6e, f).

\section{Discussion}

The interaction between population dynamics and spatial heterogeneity has been recognized both theoretically and experimentally (Rosenzweig 1981, Morris 1989, 2003, Akçakaya 2000, Keith et al. 2008), yet it remains a mostly unaddressed issue for species distribution models. While many ecological questions on species distribution are motivated by population dynamics (e.g., viability of fragmented populations, spatial management of pests, species range shifts), an explicit connection between observed distributions and dynamics is rarely pursued (Railsback et al. 2003, Guisan and Thuiller 2005, Zurell et al. 2009, Gaillard et al. 2010, McLoughlin et al. 2010, Morales et al. 2010, Mieszkowska et al. 2013). This, and other broadly recognized issues with SDMs have thus far been investigated with literature reviews, or comparative studies between existing frameworks that, in their majority, make the assumption that populations are at a state of equilibrium (Guisan and Zimmermann 2000, Araújo and Guisan 2006, Randin et al. 2006, Elith and Graham 2009, Elith and Leathwick 2009, Zurell et al. 2009, Hoffman et al. 2010, Matthiopoulos and Aarts 2010). Such comparative approaches have their utility, but as ecological practitioners we should perhaps be less concerned about which heuristic SDM performs better in particular case studies. Instead, we should simply select a sufficiently expandable inferential framework, verify its robustness (Barry and Elith 2006), and begin fleshing it out with biological mechanisms (Austin 2002, Railsback et al. 2003, Guisan and Thuiller 2005, Moorcroft and Lewis 2006, McLoughlin et al. 2010, Morales et al. 2010). Here, we have contributed to this increasingly active area of research (Tyre et al. 2001, Railsback et al. 2003, Aldridge and Boyce 2008, Gaillard et al. 2010, Hoffman et al. 2010, Morales et al. 2010, DeCesare et al. 2014).

We opted for the well-established SDM approach of HSFs (also known as resource selection functions) and developed them into population models under the familiar framework of generalized linear models. Our synthesis can lead to joint inference on spatiotemporal data, yielding parameterized population models that can be used for forecasts outside the range of observed scenarios. While sharing with other recent work (Mieszkowska et al. 2013) the motivation of confronting spatiotemporal dynamics with data empirically, our model's mechanistic component is greater and more expandable. Further, rather than requiring geo-referenced data on growth (in the form of spatial layers for survival and fecundity, e.g., Aldridge and Boyce [2008], DeCesare et al. [2014]), we fit to nonspatial population time series, such as those available from long-term monitoring studies (e.g., Sæther 1997, Gaillard et al. 1998, Brook et al. 2000, Parmesan and Yohe 2003, Stuart et al. 2004, Strayer et al. 2006).

Our work (see $A$ note on the relationship between partial fitness and habitat suitability) offers a convergent route for classic theoretical distribution modeling (e.g., optimal exploitation or ideal free distribution) and more contemporary data-driven models (SDMs). In this way, spatial and population data from different systems may unveil the extent of agreement or discrepancy between idealized theoretical models and the natural world, offering a data-driven approach to further theory development. Using simulation, we illustrated how the incorporation of robust estimates of spatial parameters (describing habitat availability and suitability) can improve our ability to predict population growth.

\section{Simplifying assumptions and routes to the framework's extension}

Currently, our approach is mostly a conceptual rather than a methodological advance, because it is applicable only within the constraints of the assumptions detailed in Ecological scope and simplifying assumptions. The work needed to relax these assumptions prescribes a complete research program for the future. For example, more explicit modeling of accessibility (see assumption 1 and Matthiopoulos [2003]), will capture source-sink dynamics as well as transient effects due to spatial colonization or behavioral movement constraints. This extension will simultaneously test the validity of assumption 2 (yearly pseudo-equilibria in species distributions) and suggest ways in which it can be relaxed.

The assumption of representative habitat use (assumption 3) constrains our method to mobile animals living in study regions within which habitat availability does not change much between the spatial ranges of individuals. If individuals are less mobile, (e.g., due to 
territoriality), then they might each experience parts of the landscape that differ in their environmental composition. Statistical estimation was effective in our numerical experiments, despite the fact that this assumption was to some extent violated by our simulation where limitations in mobility (only 12 relocations per year) meant that different animals tended to frequent different parts of the landscape. However, it is certain that the approach's performance will deteriorate if applied to instances of large-scale data in landscapes where habitat availability is spatially nonstationary. To address this, the approach would need to be extended to account for more localized definitions of habitat availability and usage.

Our decision not to model resource depletion explicitly (assumption 4), is perhaps less severe than it first appears. Although there is no methodological obstacle to extending our model to represent two-species dynamics, there may be little practical reason to do this in some cases. For example, some generalist consumers may not drive depletion in a resource, so for such species, resource data would best be provided as a covariate. Specialist consumers feeding on a fastregenerating keystone resource (e.g., plankton) might also be handled with a similar, covariate-driven approach. Specialist consumers that rapidly deplete their single resource are unlikely to present strong correlative signals with such a heavily depleted resource layer. So, although in these cases, it might make sense to model the dynamics between the consumer and the resource mechanistically, from a data perspective, it may be more practicable to use non-depletable proxies of resource productivity as habitat descriptors. More broadly, in different ecological contexts, the quantities characterized here as resources and risks for a focal species can be understood as populations of prey or predators with dynamics of their own. The exemplars presented in the present study do not account for these explicitly. However, we know of no methodology that can statistically fit spatially explicit models of multispecies population dynamics to time-series data. Our approach demonstrates how to achieve this objective by reducing spatially heterogeneous responses to (nonspatial) numerical covariates of observed population dynamics.

Models that extend our treatment of density dependence (assumption 5) to nonlinear forms, such as the Allee effect, are possible, but would need to extend the mathematical calculations in our Appendix B.

By using non-saturating, polynomial forms for the components of fitness (assumption 6), we have implicitly assumed that the population's growth can be unconstrained in the short term (specifically, that fecundity is not bounded above and can increase in proportion to resource availability). However, particularly for $K$ selected species, reproductive potential is likely to be physiologically constrained, posing the need for nonlinear responses to resources (Austin 1999, 2002). In reality, such constraints to individual performance may not have the opportunity to limit population growth because (1) local resource availability may not reach high enough levels, (2) population density dependence may limit rates of growth before physiological limits are reached, and (3) particularly for $r$-selected species, constraints to reproduction may not be very tight. However, if the non-saturating forms are violated (by, say, small populations of mammals living in rich environments), the model will tend to over-predict population growth. This can be amended by use of asymptotic response functions and replacement of the analytic results in Appendices A and B by approximations using numerical integration. Similarly, numerical integration can be used to capture responses to conditions that are not symmetric around the optimum value of suitability (Austin 1999, 2002). Due to their computational overhead, such numerical approaches should be constrained to low-dimensional $E$-spaces (i.e., case studies where the distribution and growth of populations are driven by a small number of environmental variables).

We have assumed that covariates determine partial fitness additively (assumption 7). We feel that this is a simplistic, but nevertheless suitable null model from which to begin exploring nonadditive interactions between resources. In his monograph on resource competition and community structure, Tilman (1982) presents a classification of possible interactions between two (or more) resources in determining population growth (e.g., see Fig. 5; Tilman 1982:29). Our assumption of additivity corresponds to Tilman's null model of perfect substitutability between resources. Hence, for two resources, the linear predictor for the partial fitness (or the HSF) in our framework takes the form $\beta_{0}+\beta_{1} X_{1}$ $+\beta_{2} X_{2}$. It is easy for our model to be extended to Tilman's more complicated scenarios by use of interaction terms, giving predictors of the form $\beta_{0}+\beta_{1} X_{1}+$ $\beta_{2} X_{2}+\beta_{3} X_{1} X_{2}$. Hence, for example, a positive value for $\beta_{3}$ would yield Tilman's scenario of resource complementarity, whereas a negative value would signify antagonistic resources.

Our assumption about the additivity of partial fitness in determining average fitness (assumption 8) can only be relaxed with breakthroughs in our ability to model complementarity in habitat use under different lifehistory activities (e.g., Guisan and Thuiller 2005, Wilson et al. 2012, Russell et al. 2015). Many organisms are able to survive by integrating habitat variation across space and time. For example, an animal that moves between a feeding patch and a water hole is effectively creating a sufficient habitat type from the combination of two insufficient ones. Depending on the proximity of the constituent (insufficient) habitats, and the locomotory capabilities of the animal, such complementary use may be thought of as generating availability in $E$-space for the sufficient habitat even though it may not physically exist in $G$-space. Hence, to extend our framework to 
include complementarity, we need to redefine availability, for example by devising an appropriate smoothing operation of the observed availabilities in $E$-space before the Gaussian decomposition (Parametric formulations of habitat availability) is applied. Following such a correction, the remainder of our methodology could be applied as demonstrated in the present study.

The incorporation of different aspects of population structure (assumption 9) is the next stage of biological complexity for this approach, enabling it to be more naturally applied to organisms with multiannual prerecruitment stages. Ultimately, the addition of individual variation, particularly with a view to genetic adaptation (assumption 10) will allow us to extend our operational definition of fitness to include evolution (e.g., by using elements from approaches such as integral projection models; Ellner and Rees 2006).

\section{Computational and data requirements}

Computational issues and, by implication, the expedience of analytical bridges between spatial heterogeneity and population growth, will become more important with increases in the complexity of population models. For example, with the addition of age structure, use of nonstandard/overdispersed error structures, or integrated use of different population data, our approach will need to be paired with computer-intensive estimation. This will additionally allow us to carry out simultaneous inference on both spatial and population data, unlike the three-stage approach used here.

Successful application of our framework relies on data availability. For our illustrations in Figs. 5a-d and $6 \mathrm{a}-\mathrm{d}$, we used large data sets to clearly show the differences in the quality of fit and prediction between spatial and mean-field models. With our simulation, the model's good performance was robust to gradual reductions in data availability (Appendix C). In our most severely depleted data experiment (Figs. 5e, f and $6 \mathrm{e}, \mathrm{f})$, good performance was evident despite the fact that $99.5 \%$ of the original data were removed. We found that the method will perform poorly if provided solely with data from few populations that have already reached their carrying capacities in static environments, because such data sets would contain little information on how the species responds to contrasting environments and low (as well as high) population densities. That is not to say that data from a single population will always be uninformative: a single population that has been witnessed responding to a changing environment for 10 years may be as valuable as 10 populations that have been observed over a single year at different locations of the species' range. Clearly, our idealized simulations remained close to the assumptions of our framework, and therefore the sensitivity of these results on the severity of violation of each of our assumptions will need to be investigated more extensively.

Equally, our model would be vulnerable to poorly performing HSFs (e.g., those that explain less than half of the variability in the observed spatial distribution of a species). This may be alleviated by the use of additional covariates, if data are available. Such increases in dimensionality are catered for by our approach. Statistical approaches to improving model performance and constraining complexity are well understood in the species distribution literature (HSFs using 20 or 30 candidate environmental covariates are not an unusual occurrence). The parametric decomposition routines used in Parametric formulations of habitat availability and Parameter estimation from space-use and population time-series data to approximate habitat availability are also known to be quite robust when used in highdimensional spaces. In terms of speed, because our population model-fitting uses constructed covariates that are purely algebraic (no numerical integrals), there is little computational overhead to increasing the number of covariates.

Furthermore, even though in our simulations we used counts on a spatial grid (hence the use of Poisson models in estimating the HSF parameters), the method can work equally well with other types of HSF models fitted to other types of data, e.g., grid-free, presence-only data (Warton and Shepherd 2010, Aarts et al. 2012), such as those derived from telemetry methods (Aarts et al. 2008). The intercept of the HSF estimated from presence-only data carries no biological information on population size, but our framework retrieves the intercept independently, using the population time-series data (on condition that they are not simply an index of relative population size).

The issue of data availability is important when considering our approach in the context of other work done at the interface between population distribution and demography. Much of this work has focused on modeling spatially explicit survival data (Aldridge and Boyce 2008, Gaillard et al. 2010, DeCesare et al. 2014). This is a fruitful approach and not entirely exclusive of what we have done. However, for many ecological systems, spatial data on survival will be a rather tall order. Our approach has the advantage of relying on aggregate (i.e., nonspatial) data on population growth that can be more readily obtained from population monitoring surveys. Further, it integrates the effects on fitness across multiple habitats and can thus explain local demographic responses to nonlocal (i.e., regional, or global) environmental gradients.

\section{Broader ecological impact}

The ideas presented in this study will allow further building work upon two ecological cornerstones. The first is critical habitat. It has long been argued that the observed usage of a habitat is not necessarily proportional to its contribution to fitness. Since the original study by Van Horne (1983) enumerating reasons why density is a misleading indicator of habitat quality, there have been several other studies reinforcing the argument (Garshelis 2000, Loiselle et al. 2003, Railsback et al. 
2003, Niemi and McDonald 2004). Nevertheless, spatial prioritization (e.g., for purposes of reserve design) is still mostly driven by the location of usage/abundance hotspots (Loiselle et al. 2003, Moilanen et al. 2009, Zurell et al. 2009). In this study, we have begun to develop ways to evaluate the importance of habitats while discounting the effects of habitat availability, lack of distributional optimality, and intraspecific competition. Indeed, our definition of average fitness is identical to the intuitive expression proposed by Van Horne (1983: Eq. 1).

Environmental proxies of influential covariates pose a particular problem for all statistical approaches such as ours. If animals use proxies as cues for less easily detectable covariates of fitness, we run the risk of identifying these proxies as the determinants of fitness. Arguably, a good cue is a determinant of fitness (because in its absence, the animal may not be able to detect resources or risks). Conversely, a poor proxy, or one that is readily substitutable by other cues will present a poor correlation with the observed distribution of a species and have a low chance of being mistaken as a covariate of fitness.

The second ecological cornerstone upon which future work should build is the niche. Under his biotic, abiotic, and movement (BAM) framework, Soberón (2007) identifies three determinants of observed species distributions: habitat characteristics (making up the fundamental niche), biotic interactions, and movement (giving rise to the realized niche). All three of these act in unison, and a quantitative approach that aspires to niche measurement should therefore integrate their treatment. We have begun to consider the effects of movement on fitness explicitly, and our classification of environmental dimensions into resources (e.g., prey), conditions (e.g., temperature or trace elements), and risks (e.g., predators or competitors) folds biotic and abiotic characteristics within the same model. This feature allows us to estimate the responses of the study species to all of them simultaneously, either by representing these environmental dimensions by covariate data (as we have shown), or by extending the framework to model multispecies interactions (as we have discussed). An alternative way to think about the BAM formalism is that a model that is capable of quantifying all three of the main drivers of the realized niche should also estimate one of them (the fundamental niche) when the effects of the other two are controlled for.

Older ideas about how a niche can be measured (Colwell and Fuentes 1975, Petraitis 1979, Abrams 1980, Feinsinger and Spears 1981) and more recent debates on the appropriateness of using the term in association with the outputs of SDMs (Araújo and Guisan 2006, Elith and Leathwick 2009, Soberón and Nakamura 2009, Peterson et al. 2011, Warren 2012, 2013, McInerny and Etienne 2013) have a common origin: it is genuinely difficult to quantify the niche from spatial data because it is a concept deeply rooted in current environmental space but with its branches extending into future population growth and long-term dynamics (Pulliam 2000). The working definition of a niche (e.g., Chase and Leibold 2003, Soberón and Nakamura 2009, Peterson et al. 2011) as the subset of $E$-space which allows a population to grow and persist indefinitely underlines the simple fact that SDMs never could and never will fulfill their original promise as niche models, unless their estimates are viewed in close association with their population implications. Here, we have suggested how this can be achieved. Unlike all niche-related species distribution models, our statistical approach explicitly distinguishes between the coefficients of apparent habitat suitability and the coefficients of fitness. The former are understood as environment-specific variables whereas the latter are a context-invariant property of a species during a single snapshot of its evolution.

\section{ACKNOWLEDGMENTS}

This paper is dedicated to the memory of my father, Spyros Matthiopoulos. I kept him company while working on the first half; he has kept me company ever since.

\section{Literature Cited}

Aarts, G., S. Brasseur, J. Fieberg, and J. Matthiopoulos. 2013. Quantifying the effect of habitat availability on species distributions. Journal of Animal Ecology 82:1135-1145.

Aarts, G., M. MacKenzie, B. McConnell, M. Fedak, and J. Matthiopoulos. 2008. Estimating space use and environmental preference from telemetry data. Ecography 31:140-160.

Aarts, G., J. Matthiopoulos, and J. Fieberg. 2012. Comparative interpretation of count and point methods for species distribution models. Methods in Ecology and Evolution 3:177-187.

Aarts, G. M., et al. 2014. Prey habitat model outperforms prey data in explaining grey seal distribution. 28th Annual Conference European Cetacean Society, 5-9 April 2014, Liege, Belgium.

Abrams, P. 1980. Some comments on measuring niche overlap. Ecology 61:44-49.

Akçakaya, H. R. 2000. Viability analyses with habitat-based metapopulation models. Population Ecology 42:45-53.

Aldridge, C. L., and M. Boyce. 2008. Accounting for fitness: combining survival and selection when assessing wildlifehabitat relationships. Israel Journal of Ecology \& Evolution 54:389-419.

Araújo, M. B., and A. Guisan. 2006. Five (or so) challenges for species distribution modeling. Journal of Biogeography 33:1677-1688.

Arthur, S. M., B. F. J. Manly, L. L. McDonald, and G. W. Garner. 1996. Assessing habitat selection when availability changes. Ecology 77:215-227.

Austin, M. P. 1999. A silent clash of paradigms: some inconsistencies in community ecology. Oikos 86:170-178.

Austin, M. P. 2002. Spatial prediction of species distribution: an interface between ecological theory and statistical modeling. Ecological Modelling 157:101-118.

Barry, S., and J. Elith. 2006. Error and uncertainty in habitat models. Journal of Applied Ecology 43:413-423.

Begon, M., C. R. Townsend, and J. L. Harper. 2006. Ecology: from individuals to ecosystems. Blackwell Publishing, Malden, Massachusetts, USA.

Beyer, H. L., E. Gurarie, L. Borger, M. Panzacchi, M. Basille, I. Herfindal, B. VanMoorter, S. R. Lele, and J. Matthiopoulos. 2014. You shall not pass!: quantifying barrier perme- 
ability and proximity avoidance by animals. Journal of Animal Ecology. http://dx.doi.org/10.1111/1365-2656.12275

Beyer, H. L., D. Haydon, J. Morales, J. L. Frair, M. Hebblewhite, M. Mitchell, and J. Matthiopoulos. 2010. Habitat preference: understanding use versus availability designs. Philosophical Transactions of the Royal Society B 365:2245-2254.

Boyce, M. S., and L. L. McDonald. 1999. Relating populations to habitats using resource selection functions. TREE 14:268-272.

Brook, B. W., J. J. O'Grady, A. P. Chapman, M. A. Burgman, H. R. Akcakaya, and R. Frankham. 2000. Predictive accuracy of population viability analysis in conservation biology. Nature 404:385-387.

Buckland, S. T. E., and D. A. Elston. 1993. Empirical models for the distribution of wildlife. Journal of Applied Ecology 30:478-495.

Cagnacci, F., L. Boitani, R. A. Powell, and M. S. Boyce. 2010. Animal ecology meets GPS-based radiotelemetry: a perfect storm of opportunities and challenges. Philosophical Transactions of the Royal Society B 365:2157-2162.

Chapman, J. L., and M. J. Reiss. 1999. Ecology: principles and applications. Cambridge University Press, Cambridge, UK.

Chase, J. M., and M. A. Leibold. 2003. Ecological niches: linking classical and contemporary approaches. University of Chicago Press, Chicago, Illinois, USA.

Clutton-Brock, T. H., and T. Coulson. 2002. Comparative ungulate dynamics: the devil is in the detail. Philosophical Transactions of the Royal Society B 347:1285-1298.

Clutton-Brock, T. H., O. F. Price, S. D. Albon, and P. A. Jewell. 1991. Persistent instability and population regulation in Soay sheep. Journal of Animal Ecology 60:593-608.

Codling, E. A., M. J. Plank, and S. Benhamou. 2008. Random walk models in biology. Journal of the Royal Society Interface 5:813-834.

Colwell, R. K., and E. R. Fuentes. 1975. Experimental studies of the niche. Annual Review of Ecology and Systematics 6:281-310.

Colwell, R. K., and T. F. Rangel. 2009. Hutchinson's duality: the once and future niche. Proceedings of the National Academy of Sciences USA 106:19651-19658.

Coulson, T., J. M. Gaillard, and M. Festa-Bianchet. 2005. Decomposing the variation in population growth into contribution from multiple demographic rates. Journal of Animal Ecology 74:789-801.

DeCesare, N., M. Hebblewhite, M. Bradley, D. Hervieux, and M. Musiani. 2014. Linking habitat selection and predation risk to spatial variation in survival. Journal of Animal Ecology 83:343-352.

Duchesne, T., D. Fortin, and N. Courbin. 2010. Mixed conditional logistic regression for habitat selection studies. Journal of Animal Ecology 79:548-555.

Elith, J., and C. H. Graham. 2009. Do they? How do they? WHY do they differ? On finding reasons for differing performances of species distribution models. Ecography 32:66-77.

Elith, J., and J. R. Leathwick. 2009. Species distribution models: ecological explanation and prediction across space and time. Annual Review of Ecology, Evolution, and Systematics 40:677-697.

Elith, J., S. J. Phillips, T. Hastie, M. Dudik, Y. En Chee, and C. Yates. 2011. A statistical explanation of MaxEnt for ecologists. Diversity and Distributions 17:43-57.

Ellner, S. P., and M. Rees. 2006. Integral projection models for species with complex demography. American Naturalist 167:410-428.

Emlen, J. M. 1968. Optimal choice in animals. American Naturalist 102:385-389.

Feinsinger, P., and E. E. Spears. 1981. A simple measure of niche breadth. Ecology 62:27-32.
Fisher, R. A. 1930. The genetical theory of natural selection. Claredon Press, Oxford, UK.

Fraley, C., and A. E. Raftery. 2002. Model-based clustering, discriminant analysis and density estimation. Journal of the American Statistical Association 97:611-631.

Fraley, C., A. E. Raftery, T. B. Murphy, and L. Scrucca. 2012. mclust version 4 for R: normal mixture modeling for modelbased clustering, classification, and density estimation Technical report no. 597. Department of Statistics, University of Washington, Seattle, Washington, USA.

Fretwell, S. D., and H. L. Lucas. 1970. On territorial behavior and other factors influencing habitat distribution in birds. I. Theoretical development. Acta Biotheoretica 19:16-36.

Gaillard, J. M., M. Festa-Bianchet, and N. G. Yoccoz. 1998. Population dynamics of large herbivores: variable recruitment with constant adult survival. Trends in Ecology and Evolution 13:58-63.

Gaillard, J. M., M. Festa-Bianchet, N. G. Yoccoz, A. Loison, and C. Toigo. 2000. Temporal variation in fitness components and population dynamics in large herbivores. Annual Review of Ecology and Systematics 31:367-393.

Gaillard, J. M., M. Hebblewhite, A. Loison, M. Fuller, R. Powell, M. Basille, and B. Van Moorter. 2010. Habitatperformance relationships: finding the right metric at a given spatial scale. Philosophical Transactions of the Royal Society B 365:2255-2265.

Garshelis, D. L. 2000. Delusions in habitat evaluation: measuring use, selection, and importance. Pages 111-163 in L. Boitani and T. Fuller, editors. Research techniques in animal ecology: controversies and consequences. Columbia University Press, New York, New York, USA.

Gillies, C. S., M. Hebblewhite, S. E. Nielsen, M. A. Krawchuk, C. L. Aldridge, J. L. Frair, D. J. Saher, C. E. Stevens, and C. L. Jerde. 2006. Application of random effects to the study of resource selection by animals. Journal of Animal Ecology 75:887-898.

Gillies, C. S., and C. C. St Clair. 2010. Functional responses in habitat selection by tropical birds moving through fragmented forest. Journal of Applied Ecology 47:182-190.

Godvik, I. M. R., L. E. Loe, J. O. Vik, V. Veiberg, R. Langvatn, and A. Mysterud. 2009. Temporal scales, tradeoffs, and functional responses in red deer habitat selection. Ecology 90:699-710.

Guisan, A., T. C. Edwards, Jr., and T. Hastie. 2002. Generalized linear and generalized additive models in studies of species distributions: setting the scene. Ecological Modelling 157:89-100.

Guisan, A., and W. Thuiller. 2005. Predicting species distribution: offering more than simple habitat models. Ecology Letters 8:993-1009.

Guisan, A., and N. E. Zimmermann. 2000. Predictive habitat distribution models in ecology. Ecological Modelling 135:147-186

Hall, L. S., P. R. Krausman, and M. L. Morrison. 1997. The habitat concept and a plea for standard terminology. Wildlife Society Bulletin 25:173-182.

Hebblewhite, M., and E. Merrill. 2008. Modeling wildlifehuman relationships for social species with mixed-effects resource selection models. Journal of Applied Ecology 45:834-844.

Higgins, S. I., et al. 2012. A physiological analogy of the niche for projecting the potential distribution of plants. Journal of Biogeography 39:2132-2145.

Hirzel, A. H., J. Hausser, D. Chessel, and N. Perrin. 2002. Ecological-niche factor analysis: how to compute habitatsuitability maps without absence data? Ecology 83:2027-2036.

Hirzel, A. H., and G. Le Lay. 2008. Habitat suitability modeling and niche theory. Journal of Applied Ecology 45:1372-1381. 
Hoffman, J. D., N. Aguilar-Amuchastegui, and A. Tyre. 2010. Use of simulated data from a process-based habitat model to evaluate methods for predicting species occurrence. Ecography 33:656-666.

Huston, M. A. 2002. Introductory essay: critical issues for improving predictions: Pages 7-21 in J. M. Scott, P. Heglund, M. L. Morrison, J. B. Haufler, M. G. Raphael, W. A. Wall, and F. B. Samson, editors. Predicting species occurrences: issues of accuracy and scale. Island Press, Covelo, California, USA.

Johnson, D. H. 1980. The comparison of usage and availability measurements for evaluating resource preference. Ecology 6:65-71

Kearney, M., and W. P. Porter. 2004. Mapping the fundamental niche: physiology, climate and the distribution of nocturnal lizards across Australia. Ecology 85:3119-3131.

Keith, D. A., H. R. Akçakaya, W. Thuiller, G. F. Midgley, R. G. Pearson, S. J. Philips, H. M. Regan, M. B. Araújo, and T. G. Rebelo. 2008. Predicting extinction risks under climate change: coupling stochastic population models with dynamic bioclimatic habitat models. Biology Letters 4:560-563.

Krebs, J. R., and N. B. Davies. 2012. An introduction to behavioral ecology. Fourth edition. Wiley-Blackwell, Oxford, UK

Lande, R. 1982. A quantitative genetic theory of life history evolution. Ecology 63:607-615.

Lele, S. A., E. H. Merrill, J. Keim, and M. Boyce. 2013. Selection, use, choice and occupancy: clarifying concepts in resource selection studies. Journal of Animal Ecology 82:1183-1191.

Lele, S. R., and J. L. Keim. 2006. Weighted distributions and estimation of resource selection probability functions. Ecology 87:3021-3028.

Levin, S. A. 2009. Ecology (the Princeton guide). Princeton University Press, Princeton, New Jersey, USA.

Loiselle, B. A., C. A. Howell, C. H. Graham, J. P. Goerck, T. Brooks, K. G. Smith, and P. H. Williams. 2003. Avoiding pitfalls of using species distribution models in conservation planning. Conservation Biology 17:1591-1600.

Manly, B. F. J., L. L. McDonald, D. L. Thomas, T. L. McDonald, and W. P. Erickson. 2002. Resource selection by animals: statistical analysis and design for field studies. Second edition. Kluwer Academic Publishers, Boston, Massachusetts, USA.

Matthiopoulos, J. 2003. The use of space by animals as a function of accessibility and preference. Ecological Modelling 159:239-268.

Matthiopoulos, J. 2011. How to be a quantitative ecologist: the A-to-R of green mathematics and statistics. Wiley, Chichester, UK.

Matthiopoulos, J., and G. Aarts. 2010. The spatial analysis of marine mammal abundance. Pages 68-97 in I. L. Boyd, W. D. Bowen, and S. J. Iverson, editors. Marine mammal ecology and conservation: a handbook of techniques. Oxford University Press, Oxford, UK.

Matthiopoulos, J., L. Cordes, B. Mackey, C. Duck, D. Thompson, and P. Thompson. 2014. State-space modeling reveals proximate causes of harbor seal population declines. Oecologia 174:151-162.

Matthiopoulos, J., M. Hebblewhite, G. Aarts, and J. Fieberg. 2011. Generalized functional responses for species distributions. Ecology 92:583-589.

Mauritzen, M., S. E. Belikov, A. N. Boltunov, A. E. Derocher, E. Hansen, R. A. Ims, O. Wiig, and N. Yoccoz. 2003. Functional responses in polar bear habitat selection. Oikos 100:112-124.

McArthur, R. H., and E. R. Pianka. 1966. On optimal use of a patchy environment. American Naturalist 100:603-609.

McCullagh, P., and J. A. Nelder. 1989. Generalized linear models. Second edition. Chapman \& Hall, London, UK.
McDonald, L. L., B. Manly, F. Huettmann, and W. Thogmartin. 2013. Location-only and use-availability data: analysis methods converge. Journal of Animal Ecology 82:1120-1124.

McDonald, L. L., B. F. J. Manly, and C. M. Raley. 1990. Analyzing foraging and habitat use through selection functions. Studies in Avian Biology 13:325-331.

McInerny, G. J., and R. S. Etienne. 2013. 'Niche' or 'distribution' modeling? A response to Warren. Trends in Ecology and Evolution 28:191-192.

McLoughlin, P. D., D. W. Morris, D. Fortin, E. Vander Wal, and A. L. Constantini. 2010. Considering ecological dynamics in resource selection functions. Journal of Animal Ecology 79:4-12.

Mieszkowska, N., G. Milligan, M. T. Burrows, R. Freckleton, and M. Spencer. 2013. Dynamic species distribution models from categorical survey data. Journal of Animal Ecology 82:1215-1226.

Mills, L. S. 2012. Conservation of wildlife populations: demography, genetics and management. Wiley-Blackwell, Malden, Massachusetts, USA.

Millspaugh, J. J., and J. M. Marzluff. 2001. Radio tracking and animal populations. Academic Press, San Diego, California, USA.

Moilanen, A., K. A. Wilson, and H. Possingham, editors. 2009. Spatial conservation prioritization: quantitative methods and computational tools. Oxford University Press, Oxford, UK.

Moorcroft, P. R., and M. A. Lewis. 2006. Mechanistic home range analysis. Princeton University Press, Princeton, New Jersey, USA.

Morales, J. M., P. R. Moorcroft, J. Matthiopoulos, J. Frair, J. G. Kie, R. A. Powell, E. H. Merill, and D. T. Haydon. 2010. Building the bridge between animal movements and population dynamics. Philosophical Transactions of the Royal Society B 365:2289-2301.

Moreau, G., D. Fortin, S. Couturier, and T. Duchesne. 2012. Multi-level functional responses for wildlife conservation: the case of threatened caribou in managed boreal forests. Journal of Applied Ecology 49:611-620.

Morris, D. W. 1989. Density-dependent habitat selection: testing the theory with fitness data. Evolutionary Ecology 3:80-94.

Morris, D. W. 2003. Towards an ecological synthesis: a case for habitat selection. Oecologia 136:1-13.

Murray. 1985. Population growth rate as a measure of individual fitness. Oikos 44:509-511.

Mysterud, A., and R. A. Ims. 1998. Functional responses in habitat use: availability influences relative use in trade-off situations. Ecology 79:1435-1441.

Niemi, G. J., and M. E. McDonald. 2004. Applications of ecological indicators. Annual Review of Ecology, Evolution, and Systematics 35:89-111.

Northrup, J. M., M. B. Hooten, C. R. Anderson, and G. Wittemyer. 2013. Practical guidance on characterizing availability in resource selection functions under a useavailability design. Ecology 94:1456-1463.

Nur, N. 1984. Population growth rate and natural selection. Oikos 42:413-414.

Nur, N. 1987. Population growth rate and the measurement of fitness: a critical reflection. Oikos 48:338-341.

Odling-Smee, F. J., K. Laland, and M. W. Feldman. 2003. Niche construction. The neglected process in evolution. Princeton University Press, Princeton, New Jersey, USA.

Okubo, A., and S. Levin. 2010. Diffusion and ecological problems: Modern perspectives. Second edition. Springer Verlag, New York, New York, USA.

Ollason, J. G. 1991. What is this stuff called fitness? Biology and Philosophy 6:81-92. 
Owen, D. 1980. "A table of normal integrals". Communications in Statistics: Simulation and Computation B9:389-419.

Parmesan, C., and G. Yohe. 2003. A globally coherent fingerprint of climate change impacts across natural systems. Nature 421:37-42.

Patil, G. 2002. Weighted distributions. Pages 2369-2377 in A. H. El-Shaarawi and W. Piegorsch, editors. Encyclopedia of Environmetrics. Volume 4. John Wiley \& Sons, Chichester, UK.

Patterson, T. A., L. Thomas, O. Ovaskainen, C. Wilcox, and J. Matthiopoulos. 2008. State-space models for individual animal movement. Trends in Ecology and Evolution 23:87-94.

Peterson, A. T., J. Soberón, R. G. Pearson, R. P. Anderson, E. Martínez-Meyer, M. Nakamura, and M. B. Araújo. 2011. Ecological niches and geographic distributions. Princeton University Press, Princeton, New Jersey, USA.

Petraitis, P. S. 1979. Likelihood measures of niche breadth and overlap. Ecology 60:703-710.

Phillips, S. J., R. P. Anderson, and R. E. Schapire. 2006. Maximum entropy modeling of species geographic distributions. Ecological Modelling 190:231-259.

Pinheiro, J. C., and D. M. Bates. 2000. Mixed-effects models in S and S-plus. Springer-Verlag, New York, New York, USA.

Pulliam, H. R. 2000. On the relationship between niche and distribution. Ecology Letters 3:349-361.

$\mathrm{R}$ Core Team. 2014. R: a language and environment for statistical computing. R Foundation for Statistical Computing, Vienna, Austria. http://www.r-project.org/

Railsback, S. F., H. B. Stauffer, and B. C. Harvey. 2003. What can habitat preference models tell us? Tests using a virtual trout population. Ecological Applications 13:1580-1594.

Randin, C. F., T. Dirnbock, S. Dullinger, N. E. Zimmermann, M. Zappa, and A. Guisan. 2006. Are niche-based species distribution models transferable in space? Journal of Biogeography 33:1689-1703.

Renner, I. W., and D. I. Warton. 2013. Equivalence of MAXENT and Poisson point process models for species distribution modeling in ecology. Biometrics 69:274-281.

Roff, D. A. 2008. Defining fitness in evolutionary models. Journal of Genetics 87:339-349.

Rosenzweig, M. L. 1981. A theory of habitat selection. Ecology 62:327-335.

Russell, D., E. Jones, J. Matthiopoulos, B. McClintock, P. Thompson, D. Thompson, P. Hammond, S. Moss, and B. J. McConnell. 2015. Foraging habitat preferences in two sympatric seal species. Oikos. http://dx.doi.org/10.1111/oik. 01810

Sæther, B.-E. 1997. Environmental stochasticity and population dynamics of large herbivores: a search for mechanisms. Trends in Ecology and Evolution 12:143-149.

Schick, R. S., S. R. Loarie, F. Colchero, B. D. Best, A. Boustany, D. A. Conde, P. N. Halpin, L. N. Joppa, C. M. McClellan, and J. S. Clark. 2008. Understanding movement data and movement processes: current and emerging directions. Ecology Letters 12:1338-1350.

Scott, J. M., P. Heglund, M. L. Morrison, J. B. Haufler, M. G. Raphael, W. A. Wall, and F. B. Samson, editors. 2002. Predicting species occurrences; issues of accuracy and scale. Island Press, Covelo, California, USA.

Silvertown, J. M. Franco, I. Pisanty, and A. Mendoza. 1993. Comparative plant demography: relative importance of life- cycle components to the finite range of increase in woody and herbaceous perennials. Journal of Ecology 81:465-476.

Sinclair, S. J., M. D. White, and G. R. Newell. 2010. How useful are species distribution models for managing biodiversity under future climates? Ecology and Society 15:8.

Soberón, J. 2007. Grinellian and Eltonian niches and geographic distributions of species. Ecology Letters 10:115-123.

Soberón, J., and M. Nakamura. 2009. Niches and distributional areas: concepts, methods, and assumptions. Proceedings of the National Academy of Sciences USA 106:19644-19650.

Solé, R. V., and J. Bascompte. 2006. Self-organization in complex ecosystems. Princeton University Press, Princeton, New Jersey, USA.

Stenseth, N. C. 1983. Grasses, grazers, mutualism and coevolution: a comment about handwaving in ecology. Oikos 41:152-153.

Stenseth, N. C. 1984. Fitness, population growth rate and evolution in plant-grazer systems: a repy to Nur. Oikos 42:414-415.

Stockwell, D. 2007. Niche modeling. Chapman \& Hall/CRC, Boca Raton, Florida, USA

Strayer, D. L., V. T. Eviner, J. M. Jeschke, and M. L. Pace. 2006. Understanding the long-term effects of species invasions. Trends in Ecology and Evolution 21:645-651.

Stuart, S. N., J. S. Chanson, N. A. Cox, B. E. Young, A. S. L. Rodrigues, D. L. Fischman, and R. W. Waller. 2004. Status and trends of amphibian declines and extinctions worldwide. Science 306:1783-1786

Tilman, D. 1982. Resource competition and community structure. Princeton University Press, Princeton, New Jersey, USA.

Torres, L. G., A. J. Read, and P. Halpin. 2008. Fine-scale habitat modeling of a top marine predator: Do prey data improve predictive capacity? Ecological Applications 18:1702-1717.

Turchin, P. 1998. Quantitative analysis of movement: measuring and modeling population redistribution in plants and animals. Sinauer, Sunderland, Massachusetts, USA.

Tyre, A. J., H. P. Possingham, and D. B. Lindenmayer. 2001. Inferring process from pattern: Can territory occupancy provide information about life history parameters? Ecological Applications 11:1722-1737.

Van Horne, B. 1983. Density as a misleading indicator of habitat quality. Journal of Wildlife Management 47:893-901.

Warren, D. L. 2012. In defense of 'niche' modeling. Trends in Ecology and Evolution 27:497-500.

Warren, D. L. 2013. 'Niche modeling': that uncomfortable sensation means it's working. Trends in Ecology and Evolution 4:193-194.

Warton, D. I., and L. C. Shepherd. 2010. Poisson point process models solve the 'pseudo-absence problem' for presence-only data in ecology. Annals of Applied Statistics 4:1383-1402.

Wenger, S. J., and J. D. Olden. 2012. Assessing transferability of ecological models: an underappreciated aspect of statistical validation. Methods in Ecology and Evolution 3:260-267.

Wilson, R. R., L. Gilbert-Norton, and E. M. Gese. 2012. Beyond use versus availability: behavior-explicit selection. Wildlife Biology 18:424-430.

Wood, S. N. 2006. Generalized additive models: an introduction with R. Chapman \& Hall, London, UK.

Zurell, D., F. Jeltch, C. Dormann, and B. Schröder. 2009. Static species distribution models in dynamically changing systems: how good can predictions really be? Ecography 32:733-744.

\section{Supplemental Material}

\section{Ecological Archives}

Appendices A-C and Supplement 1 are available online: http://dx.doi.org/10.1890/14-2244.1.sm 